Draft Version OCtober 27, 2018

Preprint typeset using $\mathrm{I}_{\mathrm{A}}^{\mathrm{T}} \mathrm{EX}$ style emulateapj v. 5/2/11

\title{
METALLICITY-DEPENDENT QUENCHING OF STAR FORMATION AT HIGH REDSHIFT IN SMALL GALAXIES
}

\author{
Mark R. Krumholz \\ Astronomy Department, University of California, Santa Cruz, CA 95060 \\ AND \\ Avishai Dekel \\ Racah Institute of Physics, The Hebrew University, Jerusalem 91904, Israel \\ Draft version October 27, 2018
}

\begin{abstract}
The star formation rates (SFR) of low-metallicity galaxies depend sensitively on the gas metallicity, because metals are crucial to mediating the transition from intermediate-temperature atomic gas to cold molecular gas, a necessary precursor to star formation. We study the impact of this effect on the star formation history of galaxies. We incorporate metallicity-dependent star formation and metal enrichment in a simple model that follows the evolution of a halo main progenitor. Our model shows that including the effect of metallicity leads to suppression of star formation at redshift $z>2$ in dark halos with masses $\lesssim 10^{11} M_{\odot}$, with the suppression becoming near total for halos below $\sim 10^{9.5-10}$ $M_{\odot}$. We find that at high redshift, till $z \sim 2$, the SFR cannot catch up with the gas inflow rate (IR), because the SFR is limited by the free-fall time, and because it is suppressed further by a lack of metals in small halos. As a result, in each galaxy the SFR is growing in time faster than the IR, and the integrated cosmic SFR density is rising with time. The suppressed in situ SFR at high $z$ makes the growth of stellar mass dominated by ex situ SFR, meaning stars formed in lower mass progenitor galaxies and then accreted, which implies that the specific SFR (sSFR) remains constant with time. The intensely accreted gas at high $z$ is accumulating as an atomic gas reservoir. This provides additional fuel for star formation in $10^{10}-10^{12} M_{\odot}$ halos at $z \sim 1-3$, which allows the SFR to exceed the instantaneous IR, and may enable an even higher outflow rate. At $z<1$, following the natural decline in IR with time due to the universal expansion, the SFR and sSFR are expected to drop. We specify the expected dependence of sSFR and metallicity on stellar mass and redshift. At a given $z$, and below a critical mass, these relations are predicted to be flat and rising respectively. Our model predictions qualitatively match some of the puzzling features in the observed star formation history.

Subject headings: cosmology: theory — galaxies: formation — galaxies: high-redshift — galaxies:
\end{abstract} ISM — ISM: molecules — stars: formation

\section{INTRODUCTION}

This paper attempts a first-principles theoretical analysis of metallicity-dependent star formation as a major player in determining the cosmological star-formation history. To help motivate this study, it is useful to start with a review of the observational context and the tension between these observations and current models.

The $\Lambda$ CDM cosmological model (Blumenthal et al. 1984) has been very successful in explaining the largescale structure of the universe, and the general properties of dark-matter halos in which galaxies form, including their mass distribution as a function of time and environment. While we can crudely connect the halos to observed objects via statistical inference (e.g. Conroy et al. 2006, Conroy \& Wechsler 2009), we still lack a first-principles model for determining the observable stellar content of a given dark-matter halo. Numerical simulations and semi-analytic model that attempt to do so generally encounter a number of problems.

First, the cosmic star formation rate (SFR) density is observed to be rising in time from $z>8$ till $z \sim 3$, and it remains high till $z \sim 1$ (e.g. Hopkins \& Beacom 2006

krumholz@ucolick.edu dekei@phys.nuji.ac.il
Kistler et al. 2009: Bouwens et al. 2010; Horiuchi \& Beacom 2010), while $\Lambda$ CDM predicts a strong decline of cosmological inflow rate (IR) into halos of a given mass at all epochs (e.g. Neistein et al. 2006). Since the star formation timescale is short compared to the Hubble time for most of cosmic history, the SFR and the IR are expected to be tightly locked - galaxies convert gas into stars at a rate that is ultimately determined by the gas supply rate. As a result, in most current models, the decline in IR at high $z$ drives a decline in SFR density that starts much earlier than $z \sim 1-2$ (e.g. Murali et al. 2002 Hernquist \& Springel 2003, Springel \& Hernquist 2003: Kereš et al. 2005: Baugh et al. 2005 Bouché et al. 2010: Schaye et al. 2010). There is a related observational indication for a rapid growth in SFR within a given population of halos as they grow in time, faster than the predicted mass growth by accretion till $z \sim 2$ (Papovich et al. 2010). The tension between observation and theory is emphasized by the observational indications for a "sSFR plateau", where the specific SFR (i.e. the SFR per unit stellar mass) is rather constant between $z \sim 8$ and $z \sim 1-2$, and is significantly lower than predicted at $z \geq 6$ (Weinmann et al. 2011. and references therein). The above may also be related to the "missing dwarf problem", where the low observed 
abundance of small galaxies compared to the model predictions indicates that the models tend to form stars too rapidly at high redshift (Fontanot et al. 2009, Marchesini et al. 2009, Cirasuolo et al. 2010). All the above indicate the need for a mechanism that effectively suppresses the SFR at low masses and at high redshift.

At intermediate redshifts, $z \sim 1-2$, the models seem to face an opposite problem. The observed SFR and sSFR are high, and perhaps even exceed the predicted instantaneous IR and specific IR (Daddi et al. 2007; Davé 2011; Weinmann et al. 2011). The problem is made even worse by observational indications for massive outflows from galaxies at $z \sim 2-3$ (Steidel et al. 2010, Genzel et al. 2011), which in some cases may exceed both the SFR and the predicted instantaneous IR, thus indicating that the freshly accreting gas is not the only source of gas supply.

The need to suppress SFR in small halos and at high redshift has been addressed in models by the introduction of various "feedback" mechanisms, which are assumed to inhibit star formation and/or remove gas from galaxies. Proposed mechanisms include photoionization by the UV background (see the review by Loeb \& Barkana 2001), and stellar feedback by supernovae (Dekel \& Silk 1986) or radiation pressure (Thompson et al. 2005; Murray et al. 2010, Hopkins et al. 2011). The attempts made so far to implement stellar feedback in simulations and semi-analytic models (SAM) are not really satisfactory (Springel \& Hernquist 2003, Kobayashi|2004; Cen \& Ostriker 2006; Oppenheimer \& Davé 2006; Governato et al. 2007), because they operate on scales too small to be resolved, and have to be incorporated via simplistic recipes that sometimes fail to capture the complex sub-grid physics involved. As a result, the modeling in most cases is limited to an attempt to tune the feedback prescriptions in order to obtain a better fit to the observation. Since different sub-grid prescriptions produce different results, they have limited predictive power.

The feedback mechanisms mentioned above are assumed to be effective in removing the gas from halos of virial velocity $V_{\mathrm{v}} \leq 50 \mathrm{~km} \mathrm{~s}^{-1}$. This is consistent with the indications for lack of gas in such systems at $z \sim 2-3$, based on observations of damped Lyman $\alpha$ absorption systems on the lines of sight to quasars (Barnes \& Haehnelt 2010, and references therein). However, in order to match the observed SFR history and other observational constraints, Bouché et al. (2010) have argued that the SFR has to be suppressed also in more massive halos, in the mass range $M_{\mathrm{v}} \sim 10^{10}-10^{11} M_{\odot}$, corresponding at $z \sim 2.5$ to $V_{\mathrm{v}}=50-100 \mathrm{~km} \mathrm{~s}^{-1}$. Halos in this mass range do seem to contain gas at the level of half the cosmological baryonic fraction (Barnes \& Haehnelt 2010), which calls for a non-ejective feedback mechanism. The observational indications for SFR and outflow rate that exceed the IR at $z \sim 1-3$ also call for a feedback mechanism that causes the accumulation of gas reservoirs in smaller galaxies at higher redshifts.

In this paper we consider from first principles a physical process that may offer an entirely different solution to the problems of the star formation history. In the past decade, observations of star formation in nearby galaxies have unambiguously revealed that star formation is associated with the molecular phase of a galaxy's interstellar medium (ISM) (Wong \& Blitz 2002; Kennicutt et al.
2007; Leroy et al. 2008, Bigiel et al. 2008). In gas where, averaged over kpc scales, the dominant chemical state is $\mathrm{H}$ I rather than $\mathrm{H}_{2}$, the star formation rate is more than an order of magnitude lower than in molecular gas of comparable surface density (Bigiel et al. 2010). It is likely that even in such $\mathrm{H}$ I-dominated regions star formation is only occurring in a transiently-formed molecular phase. Beyond the local universe, observations at high redshift indicate a similar phenomenon: $\mathrm{H}$ I-dominated damped Lyman- $\alpha$ systems and outer parts of Lyman break galaxies both show star formation rates more than an order of magnitude lower than would be expected for molecular gas of comparable surface densities (Wolfe \& Chen 2006 Wild et al. 2007; Rafelski et al. 2010).

Krumholz et al. (2011) show that this observation can be understood in terms of the thermodynamics of interstellar gas (also see Schaye 2004). The $\mathrm{H}$ I to $\mathrm{H}_{2}$ transition occurs in gas at high volume and column density; a high volume density produces a large $\mathrm{H}_{2}$ formation rate, while a high column density produces greater shielding against dissociating interstellar UV photons. The gas temperature depends on volume and column density in nearly the same way, because high volume densities raise the rate of cooling by collisionally-excited metal lines, while high column densities increase the amount of shielding against grain photoelectric heating. As a result, over many orders of magnitude in gas volume density, column density, and metallicity, the $\mathrm{H}$ I to $\mathrm{H}_{2}$ transition is an excellent proxy for the transition from gas that is too warm to form stars $(T \gtrsim 100 \mathrm{~K})$ and gas that is cold enough $(T \sim 10 \mathrm{~K})$ to undergo runaway collapse to stellar densities ${ }^{1}$ The subsequent numerical simulations of Glover \& Clark (2011) confirm this picture.

The distinction between the warmer atomic and colder molecular phases of the ISM is significant even in the present-day universe, but it is crucial in the early universe. The transition between the phases is highly sensitive to the metallicity of the gas, occurring at a very different column density in the Milky Way, at Solar metallicity, than in the Small Magellanic Cloud, at $\sim 20 \%$ Solar metallicity (Tumlinson et al. 2002, Bolatto et al. 2007; Leroy et al. 2007; Krumholz et al. 2009b; Bolatto et al. 2011). In low metallicity blue compact dwarf and dwarf irregular galaxies, which are thought to be local analogs of common high-redshift star-forming galaxies, H I surface densities reach $\sim 100 M_{\odot} \mathrm{pc}^{-2}$ (Fumagalli et al. 2010), whereas in Solar metallicity systems a transition to $\mathrm{H}_{2}$ generally prevents $\mathrm{H}$ I surface densities from exceeding 10-20 $M_{\odot} \mathrm{pc}^{-2}$ (Bigiel et al. 2008). Since metallicities are lower in the early universe, the metallicitydependence of the warm $\mathrm{H}$ I to cold $\mathrm{H}_{2}$ transition, and thus of the star formation rate, has a potentially dramatic effect on cosmic star formation history.

Despite these observations, only a few simulations and models of star formation over cosmological time have in-

\footnotetext{
1 A note on terminology: in many cosmological applications it is common to refer to gas at temperatures $\sim 10^{4} \mathrm{~K}$ as cold to distinguish it from hotter $\sim 10^{6} \mathrm{~K}$ halo or IGM gas. Our terminology here will be closer to that used in the ISM literature, where cold refers to gas at typical molecular cloud temperatures of $\sim 10 \mathrm{~K}$, and warm refers to typical $\mathrm{H}$ I temperatures of $\sim 10^{2}-10^{3} \mathrm{~K}$. The distinction is important because it is not possible to produce Jeans masses as low as $\sim M_{\odot}$ unless the gas can cool to $\sim 10 \mathrm{~K}$ temperatures.
} 
corporated the effects of metallicity-dependence. Analytically, Krumholz et al. (2009a) show that observations of the column density and metallicity distribution of DLAs, and their lack of star formation, can be explained by modeling the warm $\mathrm{H}$ I to cool $\mathrm{H}_{2}$ transition. On the simulation side, Robertson \& Kravtsov (2008), Gnedin et al. (2009), Gnedin \& Kravtsov (2010), and Kuhlen et al. (2011) present simulations showing that low metallicity systems, either dwarfs in the local universe or starforming galaxies at high redshift, form stars at a much lower rate than found in earlier simulations omitting metallicity effects. However, thus far these simulations have only been able to sample relatively small cosmological volumes, and have not been able to advance past the peak of the star formation history at $z \sim 2$. Thus they convey limited information about the large-scale effects of metallicity-dependent star formation. Semi-analytic models by Fu et al. (2010) and Lagos et al. (2010) have begun to explore how "metallicity-aware" star formation laws affect galaxy populations ${ }^{2}$ However, these models have numerous moving parts, and it is unclear exactly how the results depend on the numerous other parameters already built into the SAM.

In this work we present a simple toy model to understand how the need to undergo a warm $\mathrm{H}$ I to cold $\mathrm{H}_{2}$ phase transition as a prelude to star formation might affect the cosmic star formation history. Due to the numerous approximations we make, we do not expect this result to produce quantitatively exact results. Our goal is simply to understand the basic physics of the effect and its qualitative implications. The model can be summarized in three basic evolution equations for a single halo, which we present in Section 2. We then describe the results of this model in Section 3, and we compare these results to observations in Section 4. We summarize our conclusions and discuss implications in Section 5

\section{HALO EVOLUTION MODEL}

In this section we present a simple model for the evolution of the main progenitors of dark matter halos and their baryonic content. We characterize a halo by its total mass $M_{h}$ and by the gas, stellar, and metal masses $M_{g}, M_{*}$, and $M_{Z}$ in the disk galaxy that is assumed to form at the halo center. The mass of metals here includes only metals in the gas, not metals locked in stars.

\subsection{Gas and Stellar Mass Evolution}

Following the "bath-tub" model of Bouché et al. (2010), we assume that the gas and stellar mass in a halo are determined by three processes: inflow, star formation, and ejection of material by star formation feedback. The continuity equations that govern this system are

$$
\begin{aligned}
& \dot{M}_{g}=\dot{M}_{g, \text { in }}-(1-R) \dot{M}_{*, \text { form }}-\epsilon_{\text {out }} \dot{M}_{*, \text { form }} \\
& \dot{M}_{*}=\dot{M}_{*, \text { in }}+(1-R) \dot{M}_{*, \text { form }} .
\end{aligned}
$$

Here $\dot{M}_{g}$,in and $\dot{M}_{*, \text { in }}$ are the rates of cosmological inflow of gas and stars (Section 2.1.1), $\dot{M}_{* \text {,form }}$ is the star formation rate (Section 2.1.2), $R$ is the stellar return fraction,

\footnotetext{
2 Obreschkow et al. (2009) and Obreschkow \& Rawlings (2009) also studied the $\mathrm{H}$ I to $\mathrm{H}_{2}$ transition in their semi-analytic models, but they included it only in post-processing, so the star formation rate was not affected by the molecular fraction.
}

and $\epsilon_{\text {out }}$ is the amount of mass ejected into the IGM per unit mass of stars formed (Appendix A). We summarize the parameters that appear in these equations, as well as others that appear below, in Table 1. Below we discuss our estimates for the quantities appearing in these equations.

\subsubsection{Accretion rates}

The average total (baryonic plus dark matter) inflow rate into a halo of virial mass $M_{h}$ at redshift $z$ due to cosmological instreaming from the cosmic web is approximated by

$$
\dot{M}_{h, 12}=-\alpha M_{h, 12}^{1+\beta} \dot{\omega},
$$

where $M_{h, 12}=M_{h} / 10^{12} M_{\odot}, \alpha=0.628, \beta=0.14$. Here $\omega=1.68 / D(t)$ is the self-similar time variable of the EPS formalism, in which $D(t)$ is the linear fluctuation growing mode, and it is approximated by

$$
\dot{\omega}=-0.0476\left[1+z+0.093(1+z)^{-1.22}\right]^{2.5} \mathrm{Gyr}^{-1} .
$$

This approximation has been derived by Neistein et al. (2006) and Neistein \& Dekel (2008) based on EPS theory and was fine-tuned using the Millennium cosmological Nbody simulation. We adopt the cosmological parameters from WMAP: $\Omega_{m}=0.27, \Omega_{\Lambda}=0.73, h=0.7$, and $\sigma_{8}=0.813^{3}$ This expression is accurate to better than $5 \%$ for $z=0.2-5$, and to $\sim 10 \%$ for $z=0-10$. The mass dependence is accurate to $5 \%$ for halos from mass $M_{h, 12}=0.1-10^{2}$. The distribution of accretion rates about this mean can be fit by a lognormal with a standard deviation of $\sim 0.2$, plus a tail representing mergers that extends to a factor of 10 above the average (Dekel et al. 2009, Goerdt et al., 2011, in preparation), though we do not implement this scatter in our model. Although we use equation (3) for all our numerical computations, a simpler expression, which we will use to make scaling arguments later, is

$$
\dot{M}_{h} \approx 34 M_{\odot} \mathrm{yr}^{-1} M_{h, 12}^{1.14}(1+z)^{2.4} .
$$

This matches equation (3) to $\sim 10 \%$. We note that if the power 1.14 is approximated by unity, and if the 2.4 is replaced by $5 / 2$ (which is the exact predicted value in the Einstein-deSitter cosmology phase that is approximately valid at $z>1$ ), this accretion rate corresponds to a mass growth rate $M_{h} \propto \exp (-\gamma z)$, with $\gamma \simeq 0.9$.

The corresponding gas and stellar inflow rates into a halo are

$$
\begin{aligned}
& \dot{M}_{g, \text { in }}=0.17 \epsilon_{\text {in }} f_{b, 0.17} f_{g, \text { in }} \dot{M}_{h} \\
& \dot{M}_{*, \text { in }}=0.17 f_{b, 0.17}\left(1-f_{g, \text { in }}\right) \dot{M}_{h}
\end{aligned}
$$

where $f_{b}$ is the cosmic baryon fraction, $f_{b, 0.17}=$ $f_{b, \text { in }} / 0.17, f_{g, \text { in }}$ is the fraction of the inflow that reaches

3 The values of $\alpha$ and $\beta$, and the numerical coefficients in equation (4) given here have been updated to this cosmology, and are therefore slightly different than those given in Neistein et al. (2006) or Neistein \& Dekel (2008). Also note that formally equation (3) includes all accretion, both smooth and in the form of major mergers. One might therefore worry that the median accretion rate might be less than the mean given by the equation. However, since major mergers are strongly subdominant for the ranges of halo mass and redshift we be will considering, this is a relatively small effect. A more quantitative discussion of the expected level of variation in accretion rates is given in Neistein \& Dekel (2008). 
the halo as gas rather than stars, and $\epsilon_{\text {in }}$ is the fraction of accreted gas that reaches the galactic disk rather than being shock-heated and going into the galactic halo. Note that we have not attempted to include the effects of a reduction in accretion rates onto small halos with virial velocities due to suppression of cooling by the UV background after the universe is reionized. This is expected to be a large effect for halos with virial velocities below $30 \mathrm{~km} \mathrm{~s}^{-1}$, and a factor of a few effect for halos with virial velocities of $30-50 \mathrm{~km} \mathrm{~s}^{-1}$ (Thoul \& Weinberg 1996). Our omission of the effect will make some difference for the very smallest halos that we model, but the majority of the models we present below are in the regime where suppression of accretion is unimportant or is only a factor of a few effect.

In our fiducial model we compute $f_{g \text {,in }}$ using a selfconsistent approximation described in Appendix B, and we explore how this affects our results by also considering a variant model in which we take $f_{g \text {,in }}=1$ for all halos at all redshifts.

We have estimated the fraction of gas inflow from outside the virial radius that actually reaches the galaxy at the halo center, $\epsilon_{\text {in }}$, using analytic arguments and hydro cosmological simulations (Birnboim \& Dekel 2003; Kereš et al. 2005, 2009b, Dekel \& Birnboim 2006 Ocvirk et al. 2008 Dekel et al. 2009). Well below a critical halo mass $M_{h, 12} \sim 1$ there is no virial shock, and the gas flows in cold, so $\epsilon_{\text {in }} \simeq 1$. At $z>2$, in halos above the critical mass, cold streams bring in most of the gas along the dark-matter filaments of the cosmic web, and they penetrate efficiently through the hot medium, yielding $\epsilon_{\text {in }} \sim 1$ also there (Dekel et al. 2009). A statistical analysis of 400 AMR-simulated halos at $z \sim 2.5$ indeed reveals $\epsilon_{\text {in }} \sim 0.9$, with the exact value depending on how the averaging is performed (in preparation). Another estimate based on SPH simulations (Faucher-Giguere et al. 2011) gives similar values at high $z$ but somewhat smaller values, $\epsilon_{\text {in }} \sim 0.5$, at $z \sim 2.5$. At $z<2$, in halos more massive than the shock-heating scale, the cold flows are broader and their penetration power is weaker, so the accretion rate into the disk is suppressed (Dekel \& Birnboim 2006 Ocvirk et al. 2008). This turns out to explain the evolution of the red sequence of galaxies (Dekel \& Birnboim 2006 Cattaneo et al. 2006). We crudely model this using a simple analytic form following Dekel et al. (2009):

$$
\begin{aligned}
M_{12, \max } & =\max \left(2, \frac{1}{3 M_{*, \mathrm{PS}}(z)}\right) \\
\epsilon_{\text {in }} & =\left\{\begin{array}{ll}
1, & M_{h, 12}<M_{12, \max } \\
0, & M_{h, 12}>M_{12, \max }
\end{array} .\right.
\end{aligned}
$$

Here $M_{*, \mathrm{PS}}(z)$ is the Press-Schechter mass at a given redshift, which we compute from the Sheth \& Tormen (1999) ellipsoidal collapse model following the procedure outlined in Mo \& White (2002). We have also run models in which we adopt the analytic fitting formulae proposed by Faucher-Giguere et al. (2011), and found that the results are qualitatively unchanged. Of course the use of a sharp mass cutoff for accretion is an oversimplification of reality. Clearly some galaxies with masses below $M_{12 \text {,max }}$ are early types without accretion or star formation, while some with larger masses are late types that have ongoing star formation. Indeed, based on cos- mological simulations, the transition from a population of galaxies dominated by full cosmological accretion rate to a population dominated by shutdown is stretched over more than a decade in halo mass (e.g. Figure 1 of Birnboim et al. 2007, Ocvirk et al. 2008, Kereš et al. 2009a). However, given the simplicity of the remainder of our model, there would be little point in attempting to include this scatter. Moreover, given the difficulty in carrying high resolution simulations of cosmological inflow to redshifts $z \lesssim 1$, theoretical estimates of the accretion rates there must be considered highly uncertain in any event. In this paper we will be more concerned with redshfits $z \gtrsim 2$, where equation (9) may be regarded as reasonably accurate.

\subsubsection{Metallicity-Dependent Star Formation}

The model presented in the previous section is similar to that of Bouché et al. (2010). We now diverge from that model by taking into account the atomic-molecular phase transition in computing the rate at which gas turns into stars. On scales ranging from individual clouds to entire galaxies, the star formation rate is well-described by $\dot{M}_{*} \sim \epsilon_{\mathrm{ff}} M_{\mathrm{H}_{2}} / t_{\mathrm{ff}}$, where $M_{\mathrm{H}_{2}}$ is the total molecular gas mass, $t_{\mathrm{ff}}$ is the free-fall time of the gas, and $\epsilon_{\mathrm{ff}} \sim 0.01$ (Krumholz \& Tan 2007; Krumholz \& Thompson 2007). This result is an integrated version of the classical Kennicutt (1998) relation based on observations.

However galaxies at low surface densities and metallicities are observed to form stars at a rate considerably below that predicted by the Kennicutt relation (e.g. Wolfe \& Chen 2006, Wild et al. 2007; Leroy et al. 2008, Bigiel et al. 2008; Wyder et al. 2009), a result that has been successfully explained by noting that stars form only in molecular gas, and that systems with low surface density and metallicity tend to have little of their gas in molecular form (Robertson \& Kravtsov 2008; Krumholz et al. 2009a c; Gnedin et al. 2009; Gnedin \& Kravtsov 2010). We therefore adopt a star formation rate in a given halo

$$
\dot{M}_{*, \text { form }}=2 \pi \int_{0}^{\infty} f_{\mathrm{H}_{2}} \frac{\epsilon_{\mathrm{ff}}}{t_{\mathrm{ff}}} \Sigma_{g} r d r,
$$

where $\Sigma_{g}$ is the gas surface density, and $f_{\mathrm{H}_{2}}$ is the fraction of that gas in molecular form. All quantities inside the integral are functions of the distance from the disk center.

For the surface density, we assume that the gas in disks follows an exponential profile $\Sigma=\Sigma_{c} e^{-r / R_{d}}$. The scale length $R_{d}$ is assumed to be proportional to the virial radius of the halo in which it resides,

$$
R_{d}=0.05 \lambda_{0.1} R_{v},
$$

where $\lambda=(1 / \sqrt{2})\left(J / M_{h}\right) /(V R)$ is the spin parameter for a halo of angular momentum $J$, mass $M_{h}$, virial radius $R_{v}$, and circular velocity $V$ (Bullock et al. 2001), and $\lambda_{0.1}=\lambda / 0.1$. Note that we choose to make $R_{d}$ half of $\lambda R_{v}$ because for an exponential disk the half-mass radius is 1.7 scale radii, and given the uncertainties our ansatz places the half-mass radius at roughly $\lambda R_{v}$. Tidal torque theory and $\mathrm{N}$-body simulations give on average $\lambda \simeq 0.04$, but the observed radii of $z \sim 2$ galaxies (Genzel et al. 2006, 2008) suggest that the gas in them has a somewhat higher angular momentum. Following Dutton 
et al. (2010), we adopt $\lambda \simeq 0.07$ as our typical value. The virial radius is related to the virial mass and the expansion factor $a=1 /(1+z)$ by (Dekel \& Birnboim 2006)

$$
R_{v, 100} \simeq 1.03 M_{h, 12}^{1 / 3} A_{1 / 3},
$$

where $R_{v, 100}=R_{v} / 100 \mathrm{kpc}, A_{1 / 3}=A /(1 / 3)$,

$$
A=\left(\Delta_{200} \Omega_{m, 0.3} h_{0.7}^{2}\right)^{-1 / 3} a,
$$

and $\Omega_{m, 0.3}=\Omega_{m} / 0.3$. Here

$$
\Delta(a) \simeq\left(18 \pi^{2}-82 \Omega_{\Lambda}(a)-39 \Omega_{\Lambda}(a)^{2}\right) / \Omega_{m}(a)
$$

is the overdensity within the virial radius at a given epoch, $\Delta_{200}=\Delta / 200, \Omega_{m}(a)=\Omega_{m} a^{-3} /\left(\Omega_{\Lambda}+\Omega_{m} a^{-3}\right)$, and $\Omega_{\Lambda}(a)=1-\Omega_{m}(a)$. At redshifts $\gtrsim 1$, in the Einsteinde Sitter regime, $\Delta_{200} \approx 1$ and so $A \approx a$ for our standard cosmology. In this cosmology $\Delta_{200}$ grows to 1.7 at redshift 0 . Given this surface density profile, the central surface density is related to the disk gas mass by

$$
\Sigma_{c, 0}=\frac{M_{g}}{2 \pi R_{d}^{2}}=0.125 M_{g, 11} \lambda_{0.1}^{-2} M_{h, 12}^{-2 / 3} A_{1 / 3}^{-2},
$$

where $\Sigma_{c, 0}=\Sigma_{c} /\left(1 \mathrm{~g} \mathrm{~cm}^{-2}\right)$. Note that, if $M_{g} \propto M_{h}$, the surface density scales with mass and redshift like $\propto$ $M^{1 / 3}(1+z)^{2}$.

Also note that our treatment of disk sizes is extremely simple. We have ignored effects like the variation of halo concentration with mass and redshift, and the possible evolution of galactic angular momenta with redshift or other galaxy properties (e.g. Burkert et al. 2010). The disk size affects our models mainly by changing the surface density and thus the radius at which gas transitions from atomic to molecular, as discussed in the next section. This transition is also affected by metallicity, and the uncertainties associated with metallicity evolution are probably at the order of magnitude level. Given the size of these uncertainties, a more detailed treatment of disk radii seems unnecessary.

The local free-fall time depends on the gas surface density $\Sigma$ of the galactic disk. Krumholz et al. (2009c) have analyzed the structure of molecular clouds and developed a theoretical model for star formation that gives

$$
\frac{\epsilon_{\mathrm{ff}}}{t_{\mathrm{ff}}} \approx(2.6 \mathrm{Gyr})^{-1}\left\{\begin{array}{ll}
\left(\Sigma_{0} / 0.18\right)^{-0.33}, & \Sigma_{0}<0.18 \\
\left(\Sigma_{0} / 0.18\right)^{0.33}, & \Sigma_{0} \geq 0.18
\end{array},\right.
$$

where $\Sigma_{0}=\Sigma /\left(1 \mathrm{~g} \mathrm{~cm}^{-2}\right)$. The low $\Sigma$ regime corresponds to galaxies like the present-day Milky Way where molecular clouds are confined by self-gravity, while the high $\Sigma$ regime describes galaxies like low-redshift ULIRGs where they are confined by external pressure. This formula agrees well with the star formation rate observed in nearby galaxies (c.f. Figures 1 and 2 of Krumholz et al. 2009c), and we adopt it here.

Most importantly, the fraction of the ISM in the cold, molecular phase, $f_{\mathrm{H}_{2}}$, is determined by the balance between grain photoelectric heating and UV photodissociation on one hand, and collisionally-excited metal line cooling and $\mathrm{H}_{2}$ formation on dust grains on the other hand. (Near zero metallicity other processes become important, but, as we discuss below, we will not consider this regime.) Krumholz et al. (2008, 2009b) and McKee
\& Krumholz (2010) show that these processes produce a molecular fraction that depends primarily on the surface density and metallicity of the galactic disk, and relatively little on any other parameters. A crude approximation to their result is that the molecular fraction is

$$
f_{\mathrm{H}_{2}} \sim \Sigma /\left(\Sigma+10 Z_{0}^{-1} M_{\odot} \mathrm{pc}^{-2}\right),
$$

where $\Sigma$ is the total gas column density and $Z_{0}=$ $\left(M_{Z} / M_{g}\right) / Z_{\odot}$ is the metallicity normalized to the Solar neighborhood value $Z_{\odot}=0.02$. Thus regions with $\Sigma \ll 10 Z_{0}^{-1} M_{\odot} \mathrm{pc}^{-2}$ are primarily atomic, and those with $\Sigma \gg 10 Z_{0}^{-1} M_{\odot} \mathrm{pc}^{-2}$ are primarily molecular. A more accurate expression, which we adopt here, is

$$
\begin{aligned}
f_{\mathrm{H}_{2}} & = \begin{cases}1-\frac{3}{4}\left(\frac{s}{1+0.25 s}\right), & s<2 \\
0, & s \geq 2\end{cases} \\
s & =\frac{\ln \left(1+0.6 \chi+0.01 \chi^{2}\right)}{0.6 \tau_{c}} \\
\chi & =3.1 \frac{1+Z_{0}^{0.365}}{4.1} \\
\tau_{c} & =320 c Z_{0} \Sigma_{0},
\end{aligned}
$$

where $c$ is a clumping factor that accounts for smoothing of the surface density on scales larger than that of a single atomic-molecular complex. If $\Sigma$ is measured on $100 \mathrm{pc}$ scales then there is no averaging and $c \approx 1$, while on $\sim 1$ kpc scales it is $\sim 5$ (Krumholz et al. 2009c), and we adopt this as a fiducial parameter in our models. This analytic model agrees very well with numerical simulations that follow the full time-dependent chemistry of $\mathrm{H}_{2}$ formation and dissociation (Krumholz \& Gnedin 2011).

It is also possible to form stars in galaxies that are essentially free of molecules and dust, a topic that has been studied extensively since the pioneering work of Bromm et al. (2002) and Abel et al. (2002). In these cases the cooling required for star formation is driven by the tiny fraction of $\mathrm{H}_{2}$ that is able to form by gas phase processes, rather than via grain catalysis, the dominant process over most of cosmic time. However, this population III process is extremely slow and inefficient compared to the normal mode of star formation. While it is important for providing the seed metals that enable the normal mode to begin, we will neglect the contribution of population III stars to the total star formation rate of the Universe.

\subsection{Metallicity Evolution}

The final ingredient to our model is the metallicity of the gas, which affects its ability to form a cold molecular phase. The first seed metals that begin the process of $\mathrm{H}_{2}$ formation and the transition to normal star formation are produced by these population III stars. A single pairinstability supernova from one of these stars pollutes its host halo up to a metallicity of $\sim 10^{-3}$ of solar (Wise et al. 2010). We therefore adopt $Z_{\text {IGM }}=2 \times 10^{-5}$ as the fiducial "intergalactic medium" metallicity at which all halos start, and which characterizes newly accreted gas. We test our sensitivity to this choice below by also considering a model with $Z_{\text {IGM }}$ ten times higher.

Once the molecule fraction becomes non-zero and normal star formation begins, we set the metallicity based on the expected yield of the resulting stars. Following the standard practice in chemical evolution models (e.g. 


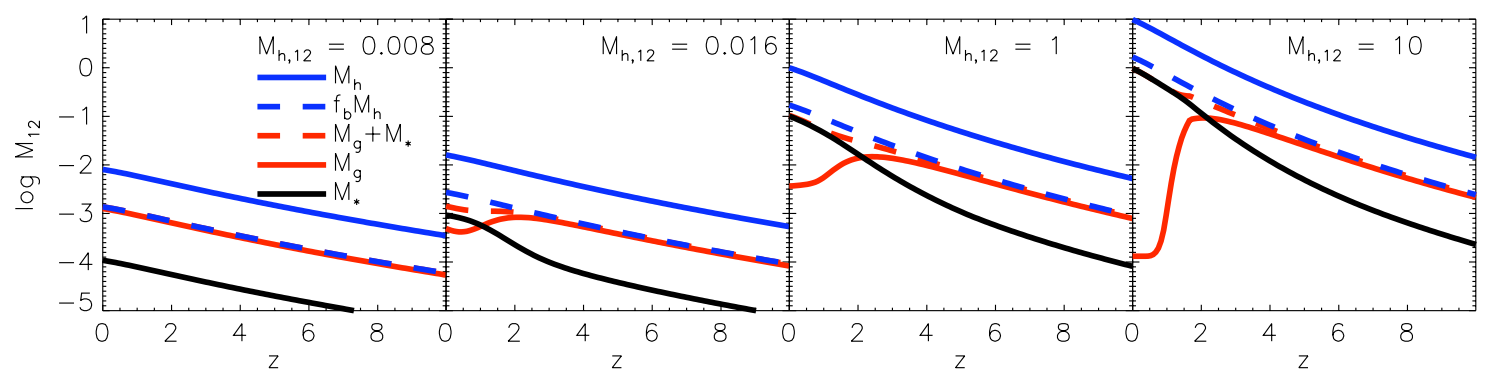

FIG. 1. - Redshift evolution of the total halo mass $M_{h}$ (solid blue lines), total baryonic mass $M_{g}+M_{*}$ (dashed red lines), gas mass $M_{g}$ (solid red lines), and stellar mass $M_{*}$ (solid black lines) for halos with present-day halo masses of $M_{h, 12}=0.008, M_{h, 12}=0.016$, $M_{h, 12}=1$, and $M_{h, 12}=10$, as indicated at the top of each panel. All masses are plotted in units of $M_{12}=M / 10^{12} M_{\odot}$. For comparison we also show the halo mass multiplied by the universal baryon fraction $f_{b}$ (dashed blue lines).

Maeder 1992), we use the instantaneous recycling approximation and write the metal production rate in terms of the yield $y$ of the stellar population. The net metal production rate corresponding to a star formation rate $\dot{M}_{*, \text { form }}$ is $y(1-R) \dot{M}_{* \text {,form }}$; we adopt $y=0.069$, as discussed in Appendix A. However, only a fraction of those metals will be retained in the galaxy rather than lost in supernova explosions. This fraction is a very sharply increasing function of halo mass, and based on a rough fit to the simulations of Mac Low \& Ferrara (1999) we take the fraction of metals ejected by supernovae to be

$$
\zeta=\zeta_{\mathrm{lo}} e^{-M_{h, 12} / M_{\mathrm{ret}}},
$$

where $M_{\text {ret }}$ is the halo mass (in units of $10^{12} M_{\odot}$ ) at which supernovae become unable to eject most of their metals, and $\zeta_{\text {lo }}$ is the fraction of metals retained in halos much smaller than $M_{\text {ret }}$. As a fiducial value, based on the simulations of Mac Low \& Ferrara (1999), we adopt $M_{\text {ret }}=0.3$. Choosing a fiducial value of $\zeta_{\text {lo }}$ is more difficult, because even if almost all metals are initially ejected from the galactic disk in low mass halos (as Mac Low \& Ferrara find), many of these will not escape the halo entirely, and will later be re-accreted - as must be the case, since halos below $M_{h, 12}=0.3$ are not completely devoid of metals. In the absence of firm theoretical guidance we adopt as a fiducial value $\zeta_{\text {lo }}=0.9$, i.e. we assume that $90 \%$ of metals will be ejected completely from small halos, while $10 \%$ will be retained or re-accreted. Below, we test the sensitivity of our results to both $M_{\text {ret }}$ and $\zeta_{\text {lo }}$ by varying these fiducial values.

The two other factors capable of changing the metal content are accretion of pristine material, which adds metals at a rate $Z_{\mathrm{IGM}} \dot{M}_{g \text {,in }}$, and expulsion of existing interstellar medium by feedback, which removes metals at a rate $\left(M_{Z} / M_{g}\right) \epsilon_{\text {out }} \dot{M}_{*, \text { form }}$. This expression assumes that the expelled gas has a metallicity equal to the mean metallicity of the galaxy. Combining metal production, accretion of pristine material, and ejection of existing interstellar material, the evolution equation for the mass of metals is

$$
\begin{aligned}
\dot{M}_{Z}= & y(1-R)(1-\zeta) \dot{M}_{*, \text { form }} \\
& +Z_{\mathrm{IGM}} \dot{M}_{g, \text { in }}-\epsilon_{\text {out }} \frac{M_{Z}}{M_{g}} \dot{M}_{*, \text { form }} .
\end{aligned}
$$

Note that we distinguish between the ejection of hot metals that are already part of a $10,000 \mathrm{~km} \mathrm{~s}^{-1} \mathrm{SN}$ shock and the driving of outflow in the cold dense ISM, the former being easier to eject and more strongly dependent on the halo mass via $\zeta$. We have verified that our results are rather insensitive to the value of $\epsilon_{\text {out }}$ and to a possible halo-mass dependence in it; for more discussion of this issue, see Appendix A.

Finally, we caution that our treatment of metals neglects the existence of metallicity gradients within galactic disks. In the local universe, these are observed to be relatively modest. For spiral galaxies the center-to-edge metallicity difference is typically at most half a dex (e.g. Oey \& Kennicutt 1993), with the metallicity gradient disappearing completely outside $R_{25}$ (e.g. Werk et al. 2011). Dwarf galaxies also have essentially no metallicity gradient (e.g. Croxall et al. 2009). Thus metallicity gradients seem unlikely to very significantly alter our results. Any potential effects are certainly likely to be smaller than those induced by changing $Z_{\text {IGM }}$ by an order of magnitude, as we do below.

\section{MODEL RESULTS}

With the model ingredients described in Section 2 in place, we can now calculate the evolution of star formation in growing main progenitor halos. The total mass, gas mass, and stellar mass then evolve according to equations (3), (1), and (2), while the metal mass evolves following equation (23). The star formation rate is given by equation (10), and the gaseous infall fraction $f_{g}$, in is calculated from equation (B4). In addition to the fiducial model, for comparison we repeat also compute several variants of the model. All model parameters are summarized in Table 1 .

In the first variant, we set $f_{\mathrm{H}_{2}}=1$ everywhere regardless of column density or metallicity. This allows us to isolate the effects of the requirement that $\mathrm{H}_{2}$ form before stars do on the star formation history of the universe. In the second, we set $f_{g \text {,in }}=1$, so that the inflow contains no stars. This allows us to understand the importance of these processes. In the third variant, we use $M_{\text {ret }}=0.03$ instead of 0.3 as the characteristic mass at which halos start retaining most of their metals. In the fourth variant we set $\zeta_{\text {lo }}=0.8$, thereby allowing halo that are below the threshold to retain $20 \%$ of their metals rather than $10 \%$ as in the fiducial model. Variants three and four allow us to test how changing our recipe for the ability of halos to retain metals, eq. 22 , alters our results. In the fifth variant we change the IGM metallicity to $Z_{\text {IGM }}=2 \times 10^{-4}$, which is 10 times the fiducial value, and corresponds to $1 \%$ of Solar. The true metal content of the IGM is likely between these extremes and probably evolves with redshift as well (e.g. Schaye et al. 2003, Simcoe 2011), but 


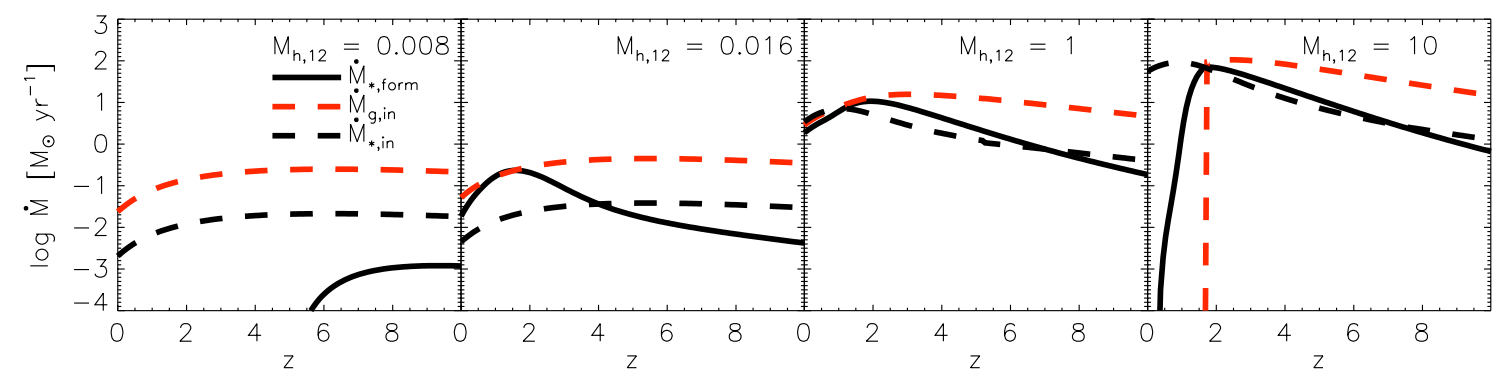

FIG. 2.- Redshift evolution of the star formation rate $\dot{M}_{*, \text { form }}$ (solid black lines), gas inflow rate $\dot{M}_{g, \text { in }}$ (dashed red lines), and stellar inflow rate $\dot{M}_{*, \text { in }}$ (dashed black lines) for the same halos as in Figure 1

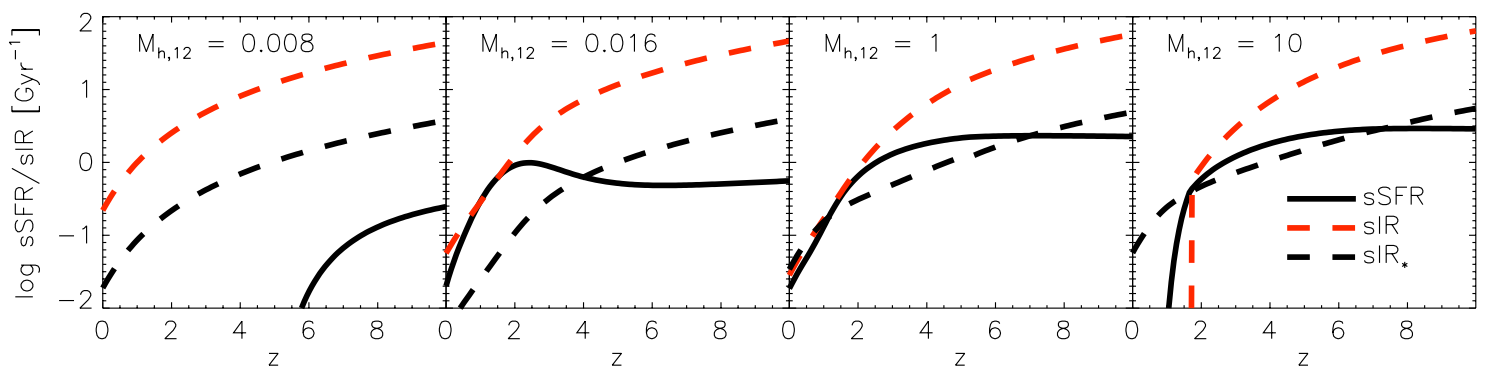

FIG. 3. - Redshift evolution of the specific star formation rate sSFR $=\dot{M}_{*, \text { form }} / M_{*}$ (solid black lines), specific gas inflow rate sIR $=$ $\dot{M}_{g, \text { in }} / M_{*}$ (dashed red lines), and specific stellar inflow rate $\operatorname{sIR}_{*}=\dot{M}_{*, \text { in }} / M_{*}$ (dashed black lines) for the same halos as in Figure 1

for simplicity we consider only these two models, which should allow us to bracket reality. Finally, in the sixth variant, which we refer to as the $\Sigma_{\mathrm{SF}}=10 M_{\odot} \mathrm{pc}^{-2}$ model, we adopt

$$
f_{\mathrm{H}_{2}}=\left\{\begin{array}{ll}
0, & \Sigma<10 M_{\odot} \mathrm{pc}^{-2} \\
1, & \Sigma \geq 10 M_{\odot} \mathrm{pc}^{-2}
\end{array},\right.
$$

i.e. where we allow star formation to occur only where the surface density exceeds $10 M_{\odot} \mathrm{pc}^{-2}$ independent of metallicity. This is very similar to the treatment of star formation adopted in many numerical simulations and semi-analytic models that do not include the physics of the atomic-molecular phase transition.

For both our fiducial model and its variants, we compute a set of 400 model halos starting at $z=$ 30 and ending at $z=0$. The halos have initial masses uniformly spaced in $\log M_{h, 12}$ from $\log M_{h, 12}=$ -5.54 to $\log M_{h, 12}=-4.06$, corresponding to presentday masses $\log M_{h, 12}=-3$ to 3 . A halo of initial mass $M_{h, \text { init }}$ begins its evolution with gas mass $M_{g, \text { init }}=0.17 f_{b, 0.17} f_{g \text {,init }} M_{h \text {,init }}$, stellar mass $M_{*, \text { init }}=$ $0.17 f_{b, 0.17}\left(1-f_{g, \text { init }}\right) M_{h, \text { init }}$, and metal mass $M_{Z}=$ $Z_{\text {IGM }} M_{g \text {,init }}$, where we set $f_{g \text {,init }}=1$ for the variant model with $f_{g \text {,in }}=1$, and $f_{g \text {,init }}=0.9$ for all other models. The choice $f_{\mathrm{g} \text {,init }}$ deserves some comment. It is clearly not realistic to assume that halos begin entirely devoid of stars $\left(f_{\mathrm{g} \text {,init }}=1\right)$, and that they form them only in the quiescent mode that our calculation includes. In mergers, or in the accretion shocks that appear when halos first begin to accrete baryons, gas surface densities can reach values much higher than our exponential disk model would predict, and these high surface densities will allow some star formation even in halos with very low metallicities. However, it is not entirely clear what value of $f_{g \text {,init }}$ we should choose to represent this effect. Our choice of 0.9 is motivated by observations indicating that stars formed in mergers account for $\sim 10 \%$ of the cosmic star formation rate budget at high $z$ (Rodighiero et al. 2011). While this contribution is obviously not the same for all halo masses, as a crude estimate we simply assume that all halos convert at least $10 \%$ of their baryons to stars early in their lives.

In Section 3.1 we present results for a few selected halos using our fiducial model, and in Section 3.2 we compare these to our variant models. We present the population statistics of our halos in Section 3.3 .

\subsection{Fiducial Model}

We consider four example halos, with present-day masses of $M_{h, 12}=0.008,0.016,1.0$, and 10.0; these masses are chosen to illustrate several interesting behaviors in the model. We note that each of these halos has a virial velocity above $25 \mathrm{~km} \mathrm{~s}^{-1}$ at all redshifts below $z \sim 10$, and above $30 \mathrm{~km} \mathrm{~s}^{-1}$ from $z=2-3$, when the bulk of the gas is accreted and most star formation occurs. Thus these halos will not be significantly affected by by ionization-driven winds (Shaviv \& Dekel 2003), though for the smallest ones the accretion rates may be reduced by a factors of a few due to reduction in cooling by the UV background (Efstathiou 1992; Thoul \& Weinberg 1996, Hoeft et al. 2006). Note, however, that below we will plot results for smaller halos for which photoionization effects are likely be important. As noted above, we have not attempted to include these simply to avoid further complicating the model.) Figures 1-4 summarize the evolutionary history of our four example halos using the fiducial model. Figure 1 shows the mass of each component, Figures 2 and 3 show the total and specific rates of star formation (including only quiescent star formation, not stars formed in accretion-driven bursts), as well as gas and stellar inflow, and Figure 4 shows the evolution of the gas-phase metallicity and column density. 


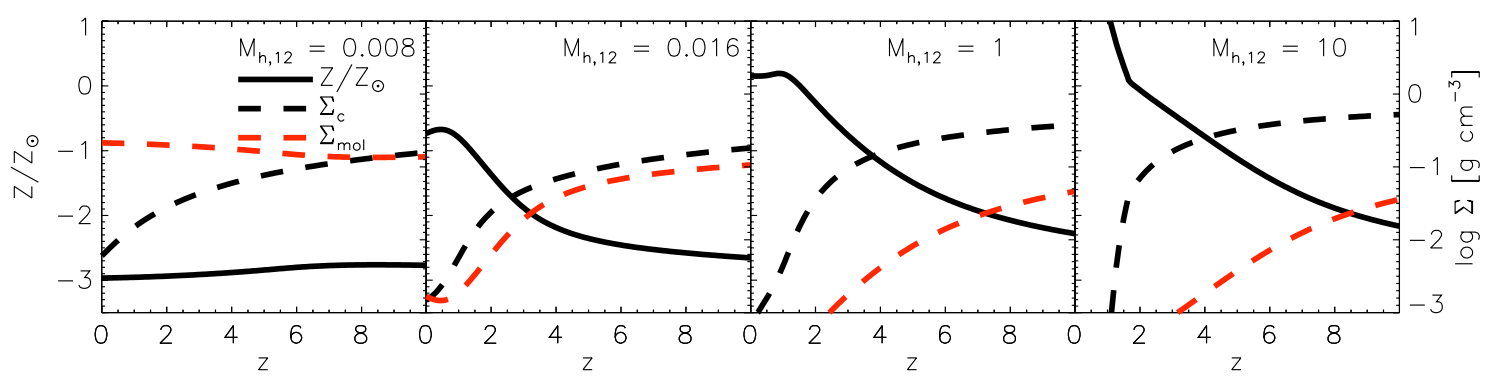

FIG. 4. - Redshift evolution of the metallicity normalized to Solar $Z / Z_{\odot}=\left(M_{Z} / M_{g}\right) / Z_{\odot}$ (solid black lines) and central column density $\Sigma_{c}=M_{g} /\left(2 \pi R_{d}^{2}\right)$ (dashed black lines) for the same halos as in Figure 1. For comparison, we also show the column density $\Sigma_{\text {mol }}$ at which a gas with the given metallicity would become $50 \%$ molecular (dashed red lines), as computed from equation 18 .

\subsubsection{Star formation cannot catch up at high redshift}

We see that, at very high redshift $(z \gg 5)$ all halos are dominated by gas rather than stellar mass, and they are forming stars more slowly than they are accreting both gas and stars from the intergalactic medium. However, there is star formation during this phase, which is critical for building up metals in the ISM. Although the halos' starting metallicities are quite low, this is compensated for by their high column densities, and as a result much of their mass is able to form molecules and participate in star formation. They remain gas-dominated simply because their star formation rate is unable to keep up with the inflow rate. To see this, note that the characteristic timescale for a halo to double its mass via inflow is (using the approximate equation 5 for the mass inflow rate)

$$
t_{\mathrm{acc}}=\frac{M_{h}}{\dot{M}_{h}}=2.1 \text { Gyr } M_{h, 12}^{0.14}(1+z)_{3}^{-2.4},
$$

where $(1+z)_{3}=(1+z) / 3$. The timescale for gas inflow to change the gas mass is similar during the era when star formation has not yet significantly reduced the gas mass. In comparison, for a halo with $M_{g}=0.17 f_{b, 0.17} f_{g} M_{h}$, where $f_{g}$ is the fraction of the baryonic mass in gas, we can estimate the time required to turn order unity of the molecular gas in a galaxy into stars by plugging $M_{g}$ into equation (15) and then equation (16). This gives a timescale for star formation at the disk center

$t_{\mathrm{SF}} \equiv \frac{t_{\mathrm{ff}}}{\epsilon_{\mathrm{ff}}}=2.5$ Gyr $\lambda_{0.1}^{0.67} f_{b, 0.17}^{-0.33} f_{g}^{-0.33} M_{h, 12}^{-0.1}(1+z)_{3}^{-0.67}$,

where we have taken $A \approx a$ and we have assumed that we are in the $\Sigma_{c, 0}>0.18$ regime; both of these approximations hold well at high $z$. Thus we see that, for $z \gg 2$, $t_{\mathrm{acc}} \ll t_{\mathrm{SF}}$, so we expect that the star formation rate will not be able to keep up with the inflow rate, more so at higher redhifts. This effect is even further enhanced by the fact that only a portion of the gas is cold and molecular. We note that even if $t_{\mathrm{SF}}$ scaled with the disk and halo crossing times, which scale with the Hubble time and thus $\propto(1+z)^{-1.5}$, the SFR was still unable to catch up with the inflow rate at high enough redshift. We should emphasize that the inability of the SFR to catch up with the IR is a result of having the disk radius given by equation (11). During a major merger the gas can be driven to a much smaller radius, leading to a shorter free-fall time and more rapid star formation. However, there are not enough major mergers to make a substantial difference in gas consumption on cosmological scales (Neistein \& Dekel 2008; Dekel et al. 2009).

\subsubsection{Metal buildup and the peak in SFR}

Despite the inability of the SFR to catch up with the IR, however, star formation does take place and build up metals. More massive halos have larger surface densities, and as a result their ratio of star formation rate and metal production rate to new gas inflow rate is higher. Furthermore, they retain a larger fraction of their metals (Equation 22). Both effects lead more massive halos build up metals more quickly than less massive ones during this phase, as seen in Fig. 4

As time passes halo surface densities drop due to the rise in disk scale lengths that accompanies expansion of the universe, and this has divergent effects on the different halos. The lowest mass halo, with a present-day mass $M_{h, 12}=0.008$, only builds up metals very slowly, and so the surface density at which it undergoes the atomic to molecular transition is relatively high. At $z \sim 8$, its central surface density is high enough to produce a $\sim 50 \%$ molecule fraction, but by $z \sim 6$ it falls too low to produce many molecules, and the star formation rate suddenly drops. This becomes self-reinforcing, since new inflow continues to bring in pristine gas, and without a continuous source of metal production within the halo the gas-phase metallicity drops back toward the IGM value. The halo effectively shuts down.

In the halo with a present-day mass $M_{h, 12}=0.016$, on the other hand, metal production is more rapid, and the galaxy remains able to form molecules and stars in its center for its entire evolutionary history. Moreover, at $z \sim 1-3$, the gap between the central surface density and the surface density required to have a $50 \%$ molecular fraction increases (see Figure 4). As a result, an increasing fraction of the disk becomes star-forming ${ }^{4}$ which allows the star formation rate to rise significantly faster than the inflow rate, until it slightly exceeds the inflow rate near $z \sim 2$. This burst of star formation at a rate above the gas inflow rate contributes to the peak in the star formation history of the universe, as we will see below.

In contrast, the two highest mass halos have higher metallicities and column densities. As they produce more and more metals, the ratio of their central surface densities to the atomic-molecular transition density rises. This allows molecules to form and star formation to take place over more and more of their area as we approach $z \sim 2$.

4 Qualitatively, such inside-out growth is a robust prediction of our model that is consistent with the star formation histories inferred for nearby galaxies using resolved stellar populations (e.g. Williams et al. 2009), but a quantitative comparison will require a more detalled model than the simple one we have computed. 
While their star formation rates are still nearly an order of magnitude below their accretion rates at $z \sim 5$, by $z \sim 2$ they have produced enough metals for their star formation rate to match their accretion rate. This effect helps suppress star formation at high $z$ and enhance the peak at $z \sim 2$.

Perhaps the most striking conclusion so far, as can be read from Figure 2 , is that our model predicts for the redshift range $z=8-2$ a steep increase with time of the SFR within each growing massive galaxy. It could be approximated by $\dot{M}_{*, \text { form }} \propto \exp (-0.65 z)$. This is very different from the assumption sometimes adopted by modelers of a SFR that is decaying exponentially with time (the "tau" model). In this redshift range, the accretion rate itself is growing slowly with time, reflecting the exponential growth of halo mass in equation 5 . The SFR is growing much more steeply, partly because $t_{\mathrm{acc}} / t_{\mathrm{SF}} \ll 1$ at high $z$ and is increasing with time, and partly because the metallicity-dependent quenching is strong at high redshift and is weakening with time (see below). By $z \sim 2$, the SFR catches up with the accretion rate as the latter has slowed down and the metallicity has become sufficiently high. As a result of the quenching of SFR at high redshift, as seen in Figure 1, gas keeps accumulating at $z>4$, and it provides additional supply for star formation at $z \sim 4-2$.

\subsubsection{Drop beyond the peak}

Past $z \sim 2$, the accretion and star formation rates in both the $M_{h, 12}=1$ and $M_{h, 12}=10$ halos drop. This drop is a result of the competition between growth in the accretion rate as halo mass increases at fixed redshift $\left(\dot{M}_{h} \propto M_{h}^{1.14}\right)$ and decline in accretion rate with redshift at fixed halo mass $\left(\dot{M}_{h} \propto(1+z)^{2.4}\right)$ as a result of the expansion of the universe. At high $z$ halos grow fast enough for the former effect to dominate, so accretion rates rises as one approaches the present. Past $z \sim 2$, however, the decline in accretion rate associated with expansion of the universe begins to dominate, and the accretion rate falls. For the $M_{h, 12}=10$ halo this decline is further accelerated when the halo reaches a mass of $M_{h, 12} \approx 2$ and gas accretion shuts off. By $z=0$ the star formation rate in this halo has dropped off from its peak value to nearly zero. In contrast, the $M_{h, 12}=1$ halo has only declined in star formation rate by a factor of $\sim 10$.

\subsection{Comparison to Variant Models}

\subsubsection{The effect of metallicity on SFR history}

In Figure 5 we compare the SFR in our fiducial model to the SFRs in our variant models. We see that the variants and the fiducial model are all very similar for the two higher mass halos. These are able to form molecules with relatively little difficulty due to their consistently high column densities, so their star formation histories are not significantly affected by metallicity-dependent star formation. The story is different for the lower mass halos. For them making the accretion purely gaseous $\left(f_{g, \text { in }}=1\right)$ or reducing the metal retention mass $\left(M_{\text {ret }}=0.03\right)$ still have relatively modest effects - the star formation lasts slightly longer in the $M_{h, 12}=0.008$ halo, and peaks slightly sooner in the $M_{h, 12}=0.016$ one - but qualitatively there is little difference. In contrast, assuming that gas can form stars regardless of whether it is in a cold, molecular phase $\left(f_{\mathrm{H}_{2}}=1\right)$, that this phase transition occurs at a metallicity-independent threshold of $\Sigma_{\mathrm{SF}}=10 M_{\odot} \mathrm{pc}^{-2}$, decreasing the maximum fraction of metals from supernovae that are ejected $\left(\zeta_{\text {lo }}=0.8\right)$, or increasing the IGM metallicity $\left(Z_{\mathrm{IGM}}=2 \times 10^{-4}\right)$ have dramatic effects in these small halos. All these changes allow much more rapid star formation at high $z$, reduce the star formation peak behavior at $z \sim 1-2$ in the $M_{h, 12}=0.016$ halo, and allow star formation to continue up to the present day in the $M_{h, 12}=0.008$ halo. Thus we see that including a realistic treatment of ISM chemistry and thermodynamics in models is likely to have a dramatic effect on the star formation history of the universe, and to make star formation sensitive to exactly how metals are distributed in halos, while changing the stellar inflow fraction or the rate of star formation in accretion shocks has relatively little effect.

The insensitivity of our results to the choice of $M_{\text {ret }}$ might at first seem somewhat surprising, given how dramatically metallicity-dependent star formation changes the halos' SF history. However, note that the halos where metallicity makes a difference have masses much smaller than $M_{\text {ret }}$ even if we reduce $M_{\text {ret }}$ by a factor of 10 compared to Mac Low \& Ferrara's value. This suggests that what matters in determining the mass below which SF will be suppressed is not the precise mass at which halos start retaining most of their metals, but instead the ability of halos below this retention mass to build up a non-negligible level of metallicity, which in turn depends on the ratio of metal production rate to accretion rate in these halos. This is a function of the star formation rate, the yield, the metallicity of the accreting gas, and the fraction of metals that are retained in small halos 5

\subsubsection{The origin of a $s S F R$ plateau}

In Figure6, we show the specific star formation rate for our fiducial model and for all the variants. Here we see that setting $f_{g \text {,in }}=1$ has a major effect on the results. It is easiest to understand this by focusing on the two higher mass halos, since for these both the fiducial model and the variants have about the same total star formation rate. We find that the fiducial model and all the variant models except $f_{g \text {,in }}=1$ have specific star formation rates in the two higher mass halos that are relatively flat at high $z$. This is fairly easy to understand. At redshifts above $z \sim 4-5$, in all of these models the stellar accretion rate exceeds the in situ star formation rate (c.f. Figures 2 and 3). By examining where in Figure 1 the stellar mass curve begins to deviate from simply tracking the total baryonic mass, one can see that stars formed in situ do not dominate the total stellar mass until $z \sim 3$. Thus, at $z \gtrsim 3$, the stellar mass in a halo of mass $M_{h}$ is approximately $M_{*}=0.17 f_{b, 0.17}\left(1-f_{g, \text { in }}\right) M_{h}$. Assuming the gas mass is $M_{g}=0.17 f_{b, 0.17} f_{g, \text { in }} M_{h}$ (i.e. that gas has not been significantly depleted by star formation yet), we therefore have a specific star formation rate

$$
\mathrm{sSFR}=\frac{\dot{M}_{*, \text { form }}}{M_{*}}=\frac{f_{g, \text { in }}}{1-f_{g, \text { in }}}\left\langle f_{\mathrm{H}_{2}} \frac{\epsilon_{\mathrm{ff}}}{t_{\mathrm{ff}}}\right\rangle,
$$

5 Although we have not directly investigated how varying the yield might change our results, note that the yield $y$ enters the evolution equations only through the combination $y(1-\zeta)$. Thus changing the yield should be nearly equivalent to changing $\zeta_{\text {lo }}$, and the yield is uncertain only at the tens of percent level, as compared to factors of order unity for the factor $1-\zeta$. 


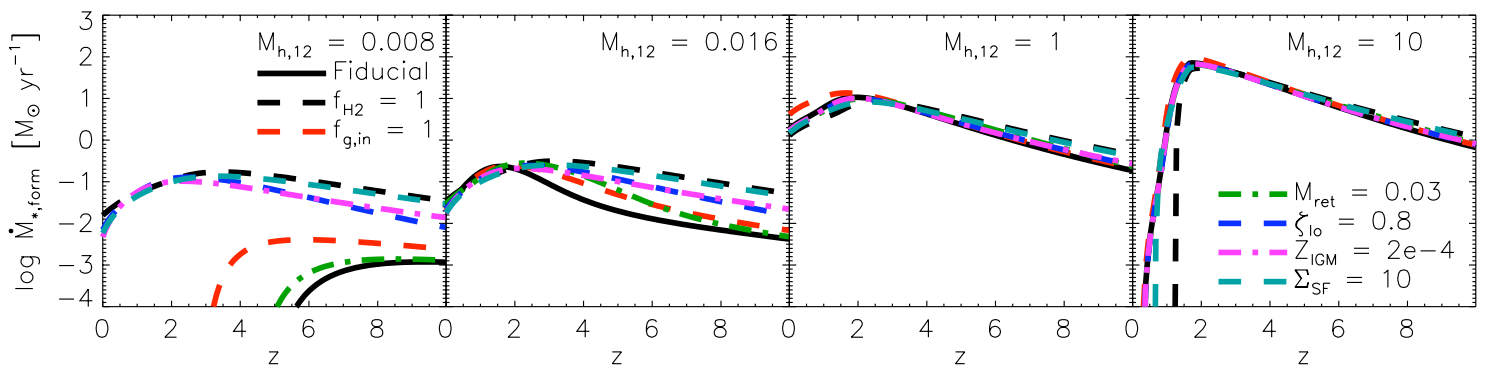

FIG. 5. - Redshift evolution of the star formation rate $\dot{M}_{*, \text { form }}$ in the same halos as in Figure 1 for our fiducial model (solid black lines), for our variant models: $f_{\mathrm{H}_{2}}=1$ (dashed black lines), $f_{g, \text { in }}=1$ (dashed red lines), $M_{\mathrm{ret}}=0.03$ (dot-dashed green lines) $\zeta_{\text {lo }}=0.8($ dashed blue lines), $Z_{\mathrm{IGM}}=2 \times 10^{-4}$ (dot-dashed purple lines), and $\Sigma_{\mathrm{SF}}=10 \mathrm{M}_{\odot} \mathrm{pc}^{-2}$ (dashed aqua lines).

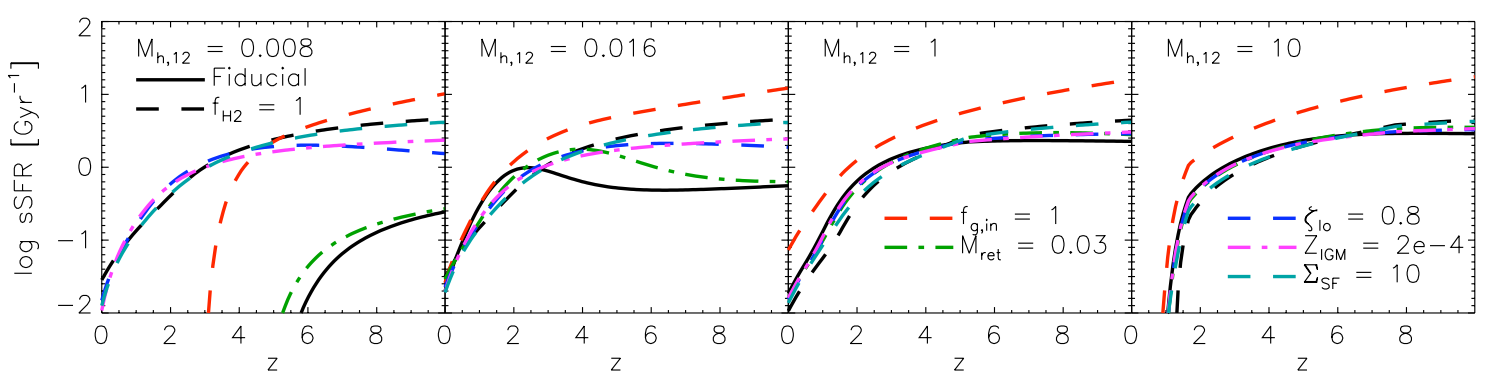

FIG. 6. - Redshift evolution of the specific star formation rate sSFR $=\dot{M}_{* \text {,form }} / M_{*}$ in the same halos as in Figure 1 Lines represent the same models as in Figure 5

where the angle brackets indicate a surface densityweighted average over the disk. Since the latter is generally $\sim(2-3 \mathrm{Gyr})^{-1}$, we obtain a nearly constant specific star formation rate of $1-3 \mathrm{Gyr}^{-1}$ at high redshift. The emergence of a sSFR plateau at high redshifts is insensitive to the exact value of $f_{g, \text { in }}$, as long as the fraction of ex situ stars is non-negligible, but it affects the amplitude of the sSFR plateau, and its extent toward lower redshifts. Metallicity-dependent star formation flattens the sSFR as a function of $z$ even further, because it makes high $z$ halos even more dominated by accreted / accretion shock-formed stars than in the model with $f_{\mathrm{H}_{2}}=1$. In contrast, in the models with $f_{g \text {,in }}=1$ halos are dominated by in situ stars at all epochs and masses. As a result, $M_{*}$ is very small at high $z$, giving rise to a larger specific star formation rate. Since $t_{\mathrm{SF}}$ gets close to $t_{\mathrm{acc}}$ as we approach the present day, $M_{*}$ rises faster than $M_{h}$, and the specific star formation rate declines toward the present, rather than remaining flat.

At intermediate redshifts, $z \sim 2-4$, where the in-situ SFR is already a major contributor to the stellar mass, the fact that the SFR is growing roughly exponentially with time leads to a similar exponential growth of its integral, the stellar mass, and thus helps extending the plateau toward $z \sim 2$.

\subsection{Population Statistics}

We now turn our attention to the ensemble properties of our entire model grid. Figure 7 shows the star formation rates and gas accretion rates in the halos as they evolve in mass with redshift. To help interpret this plot, in Figure 8 we show two ratios:

$$
s_{\mathrm{H}_{2}} \equiv \frac{\dot{M}_{*, \text { fiducial }}}{\dot{M}_{*, f_{\mathrm{H}_{2}}=1}}
$$

$$
s_{\text {IR }} \equiv \frac{\dot{M}_{*, \text { fiducial }}}{\dot{M}_{g, \text { in ,fiducial }}} .
$$

The first of these describes the factor by which the SFR is reduced by our inclusion of metallicity quenching relative to a model that does not include. The second describes the factor by which the SFR is suppressed relative to the rate of gas inflow in our fiducial model.

These plots show that a star formation law in which stars form only in cold molecular gas dramatically suppresses star formation in low mass halos. Notice that contours of constant SFR and large $s_{\mathrm{H}_{2}}$ are close to horizontal at $z \gtrsim 2$, indicating that metallicity quenching acts much like a halo mass threshold for star formation at $z \gtrsim 2$. The value of this threshold mass is sensitive to how well small halos are able to retain their metals and to the metallicity of accreting IGM gas, changing from $\sim 10^{10} M_{\odot}$ in our fiducial model to $\sim 10^{9} M_{\odot}$ in our variant where small halos retain twice as much of their metal production, and to slightly smaller masses in the model where the IGM metallicity is raised to $1 \%$ of Solar. As we will see below, this induces a $\sim 0.5$ dex shift in the total star formation rate budget of the universe at high $z$. In contrast, both the gas inflow rate and the star formation in the model where stars form equally well in warm atomic or cold molecular gas $\left(f_{\mathrm{H}_{2}}=1\right)$ or where the atomic-molecular transition is taken to be metallicity- independent $\left(\Sigma_{\mathrm{SF}}=10 M_{\odot} \mathrm{pc}^{-2}\right)$ continue all the way down to the smallest halos in our model set. For halos above the threshold, the story is quite different. At $z \gtrsim 4$, metallicity quenching reduces the SFR in these halos too, but only by factors of $s_{\mathrm{H}_{2}} \sim 2$. In contrast, the SFR lags the gas inflow rate by factors of $s_{\mathrm{IR}} \sim 3-10$, simply because star formation cannot keep up with the inflow rate. Conversely, at $z \lesssim 4$ we find that $s_{\mathrm{H}_{2}}<1$ in massive halos, indicating that star formation rates are 


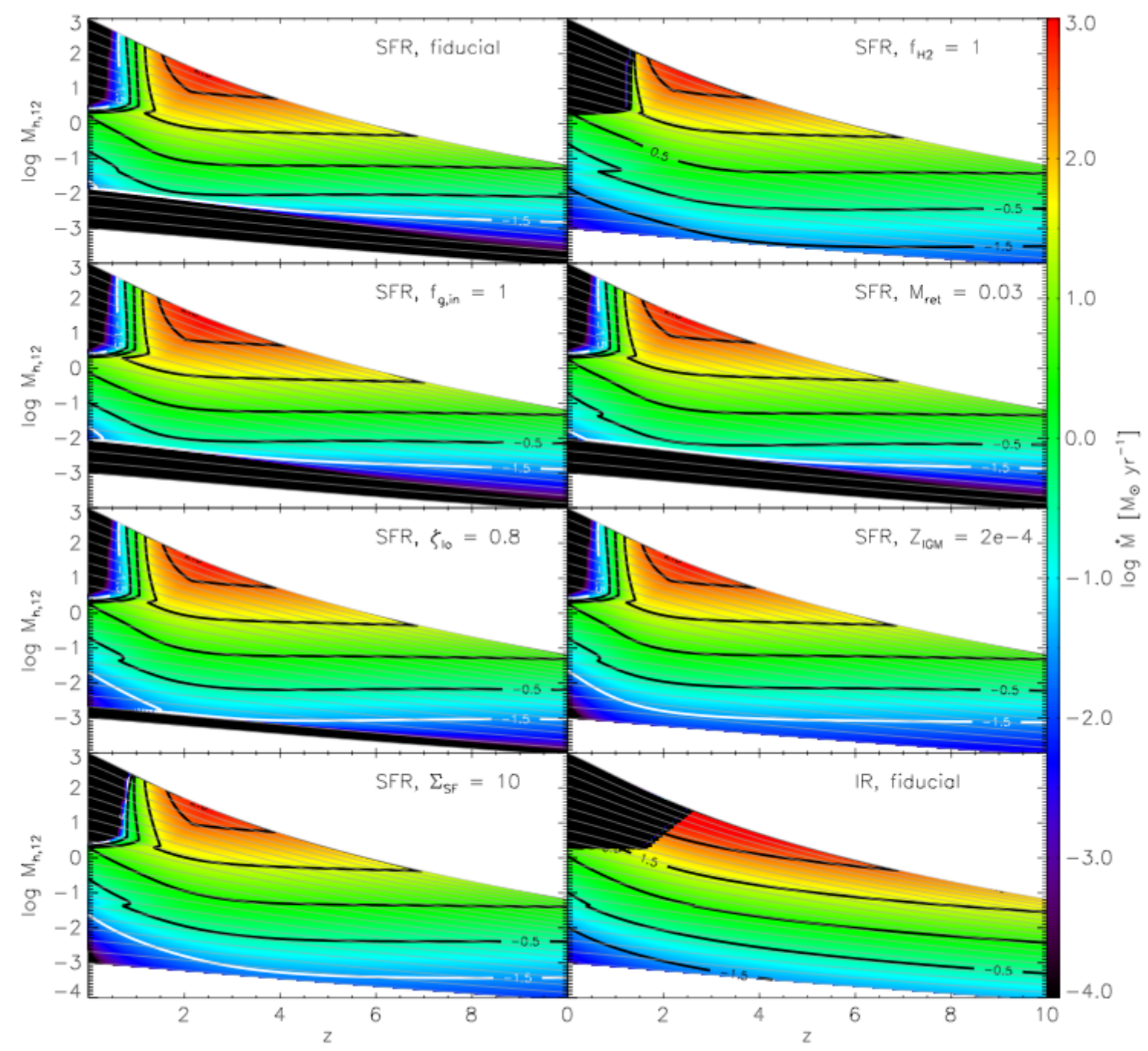

FIG. 7. - Star formation rate (SFR) and gas inflow rate (IR) as a function of halo mass and redshift. The rates are indicated by color, and are limited to our model grid. Heavy white or black lines are contours of constant SFR or IR, $\operatorname{running} \operatorname{from} \log \left(\dot{M} / M_{\odot} \mathrm{yr}^{-1}\right)=-1.5$ to 2.5 in steps of 1 dex, as indicated. Light gray lines indicate the mass versus redshift for 20 sample halos in our grid of 400 . The panels show the SFR in our fiducial model and in our variant models and the IR in our fiducial model, as indicated.

actually higher in the fiducial model than in the $f_{\mathrm{H}_{2}}=1$ or $\Sigma_{\mathrm{SF}}=10 M_{\odot} \mathrm{pc}^{-2}$ models. The reason for this behavior is that metallicity quenching reduces the SFR at high $z$ when the metallicity is low, but it does not remove the gas. At lower $z$ when the metallicity rises, this gas is able to form stars. Thus metallicity quenching in large halos simply delays star formation, helping to produce the peak of cosmic SFR at $z \sim 2$. In contrast, the choice of $f_{g \text {,in }}$ or $M_{\text {ret }}=0.03$ clearly makes little difference. We therefore conclude that the star formation history of the universe is sensitive to the need to form a cold phase, and that the ability of different halos to do so depends on their ability to retain metals. The SF history of the universe does not depend strongly on stars being formed externally or in accretion shocks or on the exact mass at which halos become efficient at retaining metals.

Figure 9 shows the corresponding specific star formation and specific inflow rates. We see that the fiducial model exhibits a very wide range of halos masses and redshifts over which the specific star formation rate is $\sim 2 \mathrm{Gyr}^{-1}$. At lower $z$ the specific star formation rate starts to fall, as star formation both increases the stellar mass and decreases the gas mass, but this process takes some time to complete, and in most halos the specific star formation rate does not drop below $1 \mathrm{Gyr}^{-1}$ until after $z=2$, and does not fall below $0.5 \mathrm{Gyr}^{-1}$ until $z \sim 1$. The model with $f_{\mathrm{H}_{2}}=1$ exhibits a slightly larger range of specific star formation rates, but except for very low mass halos, it is qualitatively similar to the fiducial model. Thus we conclude that molecules do not dramatically change the specific star formation rate in massive halos.

In contrast, the model with $f_{g \text {,in }}=1$ reaches sSFRs of many tens per Gyr, and the specific inflow rate reaches similarly large values. This is the same effect we identified in the previous section in studying the histories of individual halos. When $f_{g \text {,in }} \neq 1$, halos at very high $z$ tend to be dominated by stars that are directly accreted or formed in accretion shocks, so their stellar mass simply scales with the halo mass. Since the star formation 


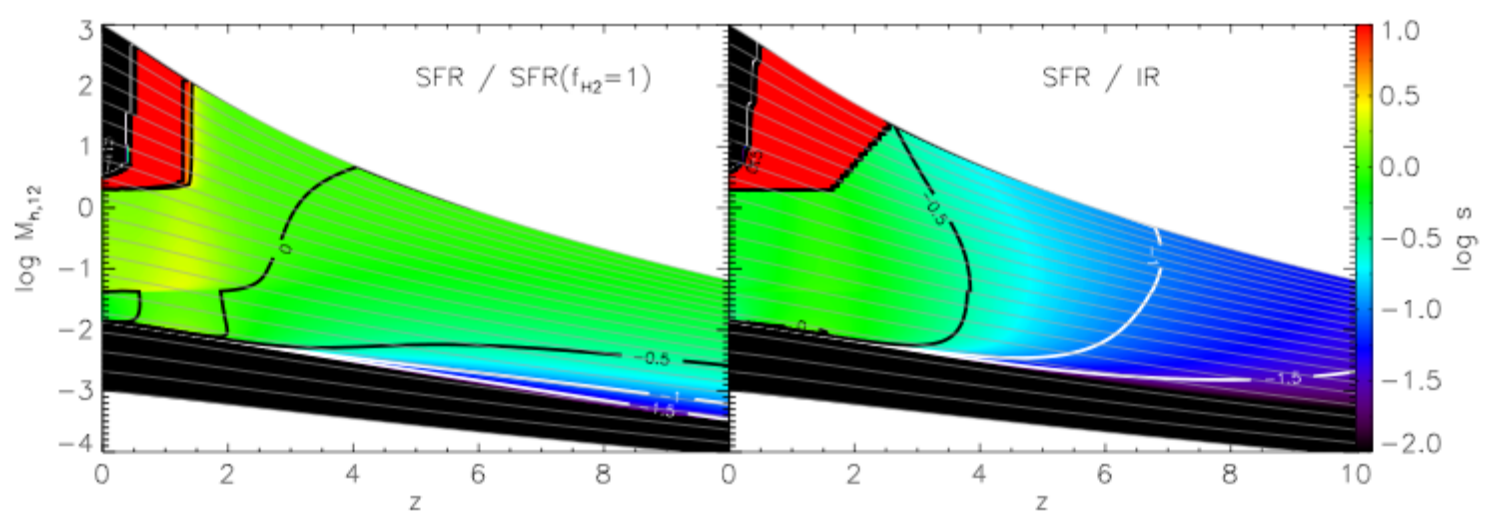

FIG. 8. - SFR suppression factor in our fiducial model relative to the model without metallicity quenching (left) and relative to the inflow rate (right). The format is similar to Figure 7 but here colors indicate the factor $s_{\mathrm{H}_{2}}$ (equation 28) or $s_{\mathrm{IR}}$ (equation 29). The former is the factor by which metallicity quenching suppresses star formation, and the latter is the ratio by which the SFR is smaller than the gas inflow rate in our fiducial model. Contours indicate values of $\log s=-1.5,-1,-0.5,0$, and 0.5 as indicated, i.e. suppression factors of $0.032,0.1,0.32,1$, and 3.2. Note that $s>1$ corresponds to an enhancement of the star formation rate rather than a suppression. Also note that in the upper left of the plot (high $M_{h}$, low $z$ ) the SFR and IR in all the models become very small, and as the result the value of $s$ is set by numerical noise in the integrator. To suppress this effect in this plot we set $s=0$ wherever the fiducial model SFR is below $10^{-3} M_{\odot} \mathrm{yr}^{-1}$.

rate scales with the gas mass (and thus also with the halo mass), the specific star formation rate is constant. For $f_{g \text {,in }}=1$, on the other hand the stellar mass is no longer proportional to the halo mass at high $z$, and the sSFR becomes large.

It is interesting to notice the contrasting roles played by metallicity quenching (i.e. the fact that $f_{\mathrm{H}_{2}} \neq 1$ ) and stellar inflow (i.e. the fact that $f_{g \text {,in }} \neq 1$ ). In order to have a SFR that increases with time but a sSFR that is flat, as the observations discussed in Section 1 appear to demand, one requires that the SFR deviate from the inflow rate, but that the total stellar mass does follow the time-integrated inflow rate. These seemingly contradictory requirements are met by a combination of suppression of star formation by metallicity quenching and increase in stellar mass due to stellar inflow.

\section{COMPARISON TO OBSERVATIONS}

In this section we use our model grid of 400 halos to generate predictions for a number of observed correlations between various properties of galaxies that have been reported in the literature, or that will become observable in the next few years as new facilities come online. Unless stated otherwise, all the comparisons in this section use our fiducial model.

\subsection{SFR in a growing galaxy}

We can also use our models to predict the evolution of SFR or other galaxy properties with observational samples selected in a variety of ways. One observational approach is to select galaxies based on their comoving number density. In this strategy, at each redshift $z$ one selects galaxies with luminosities $L$ near a cutoff luminosity $L(z)$ chosen based on the condition that the comoving number density of galaxies with luminosities $L>L(z)$ satisfy the condition that $n(>L(z))=n_{0}$, where $n_{0}$ is some fixed number density. This approach is useful because the comoving number density of main halo progenitors should, to good approximation, remain constant with redshift. Thus this technique should produce a selection that is close to following one of our theoretical main progenitor tracks in Figure 7. It is therefore a straightforward prediction of our model.

To compare our models to samples of this sort, we first compute the halo abundance $n\left(M_{h}, z\right)$, the number density of halos at redshift $z$ with masses in the range $M_{h}$ to $M_{h}+d M_{h}$, using the Sheth \& Tormen (1999) approximation, following the computation procedure outlined by Mo \& White (2002). At each $z$, we then numerically solve for the halo mass $M_{h}(z)$ for which $\int_{M_{h}(z)}^{\infty} n\left(M_{h}, z\right) d M_{h}=n_{0}$, where $n_{0}$ is the target number density. We then interpolate between the two model halos in our grid that most closely bracket $M_{h}(z){ }^{6}$

In Figures 10 and 11 we compare the SFR and stellar mass as a function of lookback time for our model halos to three number density-selected samples, taken from Marchesini et al. (2009), Papovich et al. (2011), and Lundgren et al. (2012, in preparation). 'The observational samples use a threshold density $n_{0, \mathrm{obs}}=2 \times 10^{-4}$ $\mathrm{Mpc}^{-3}$ for Papovich et al. and Marchesini et al., and $1.8 \times 10^{-4} \mathrm{Mpc}^{-3}$ for Lundgren et al. For the latter sample, only stellar masses are available, not star formation rates. The choice of $n_{0}$ for the theoretical comparison requires some care, because depending on the selection method the sample is likely to be at least somewhat incomplete. Halos at a given mass will show some scatter in their luminosity, and downward scatter (e.g. a low UV luminosity associated with a temporarily low SFR) will remove galaxies from the sample. Upward scatter can also add lower mass galaxies to the sample, but the effects are not symmetric because, if star formation histories are bursty, then galaxies will tend to spend more time with SFRs below their long-term average than with SFRs above their average. van Dokkum et al. (2008) estimate that $\sim 50 \%$ of galaxies at $z \sim 2$ will have suppressed SFRs, so that the appropriate value of $n_{0}$ for a UV-selected sample such as that of Papovich et al. is a

6 In deriving our comparison from the observational grid in this manner, we implicitly assume that luminosity or stellar mass (which is used to derive the number density in the observed sample) is a monotonically increasing function of halo mass. This assumption is satisfied by our model grid, at least over the redshift ranges where luminosity or stellar mass selection are used for the observations. 


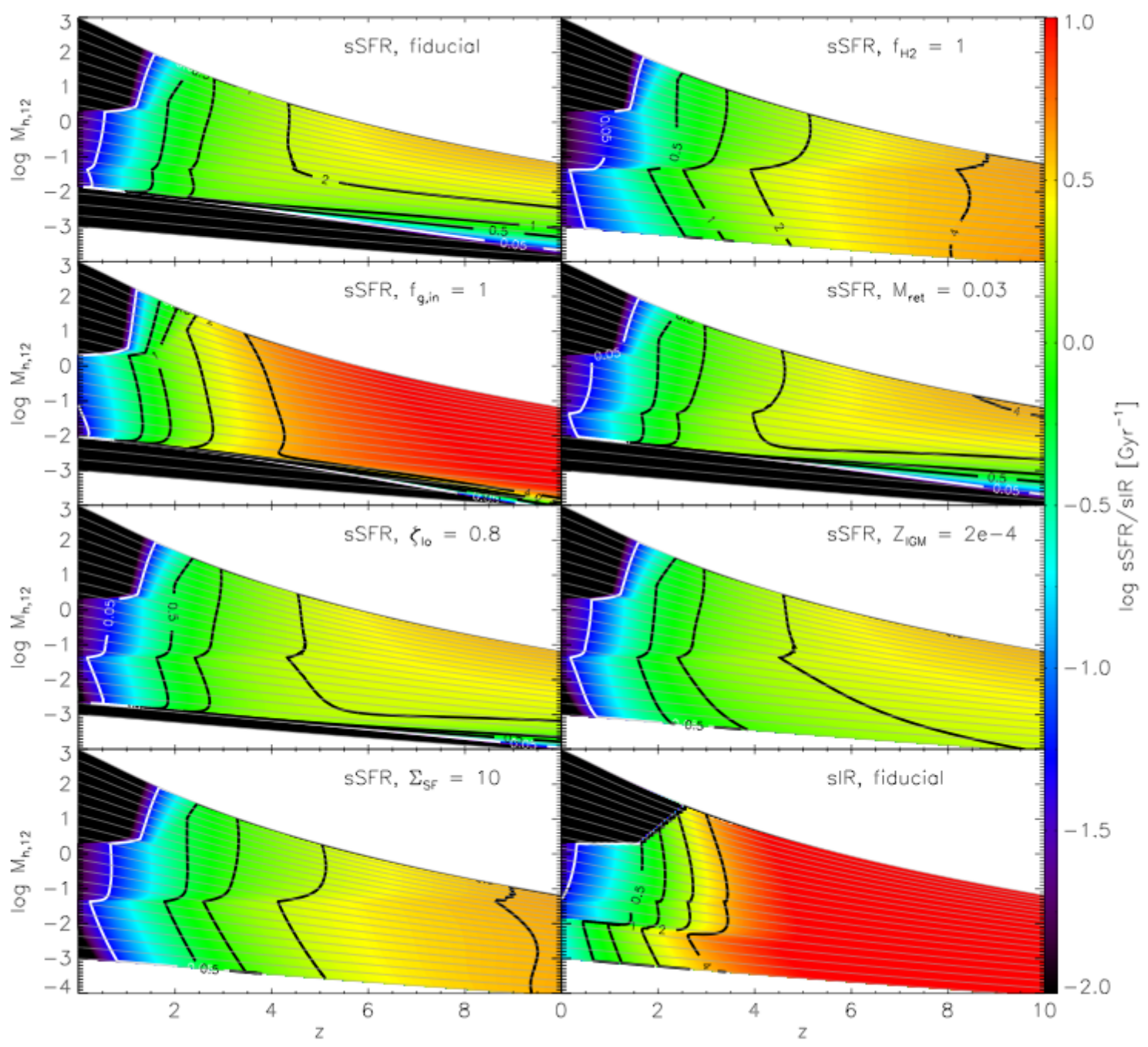

FIG. 9. - Specific star formation rate and gas inflow rate as a function of halo mass and redshift. This is the same as Figure 7 but here colors indicate specific rather than total star formation rate or inflow rates. The heavy white and black contours indicate specific star formation or gas inflow rates of sSFR or $\mathrm{sIR}=0.05,0.5,1,2$, and $4 \mathrm{Gyr}^{-1}$, as indicated.

factor of $\sim 2$ above the nominal value. The samples of Marchesini et al. are stellar mass selected and those of Lundgren et al. are H-band selected, and should therefore be substantially more complete; for these samples the appropriate value of $n_{0}$ to choice is probably close to the nominal one for the survey. Thus we plot curves for our model galaxies selected using $n_{0, \text { model }}=2 \times 10^{-4}$, $4 \times 10^{-4}$, and $8 \times 10^{-4} \mathrm{Mpc}^{-3}$, corresponding to perfect completeness, factor of 2 incompleteness, and factor of 4 incompleteness in the observations. As the figures show, for reasonable estimates of observational completeness, the models do a good job reproducing the observed trends in SFR and stellar mass. In particular, we see that in both the models and the data, SFRs and stellar masses rise roughly exponentially with $-z$ at high $z$ before leveling off near $z \approx 2-3$.

In fact, the evolution of SFR and stellar mass in the model halo with $M_{12,0}=10$ shown in Figures 1 and 2 . which has a halo mass of $10^{11} M_{\odot}$ at $z \sim 6$, is very similar to the observed evolution seen in Figures 10 and 11.

\subsection{Evolution of sSFR at Fixed Stellar Mass}

Another common observational method is to examine the properties of galaxies at fixed stellar mass across a range in redshift (e.g. Papovich et al. 2006; Stark et al. 2009: González et al. 2010). To compare to observations of this sort, we pick a target stellar mass in the range $\sim 10^{9}-10^{11} M_{\odot}$ for which high-redshift observations are available, and at each $z$ in our model grid we select the halo with stellar mass closest to the target value. We then plot the corresponding sSFR in Figure 12 For comparison, we also plot a region illustrating the range of observationally-determined sSFR values based on a data compilation from Weinmann et al. (2011). We see the same general result as in Figure 9] in our fiducial model the sSFR has a nearly constant value of $\sim 2$ $\mathrm{Gyr}^{-1}$ from $z \sim 8$ to $z \sim 2$. This is in good agreement with the observations. In contrast, the specific gas infall rate sIR $=\dot{M}_{g \text {,in }} / M_{*}$ in these halos, and the specific star formation rate if $f_{g \text {,in }}=1$, is more than an order of magnitude higher at $z \sim 8$ than at $z \sim 2$, in disagree- 


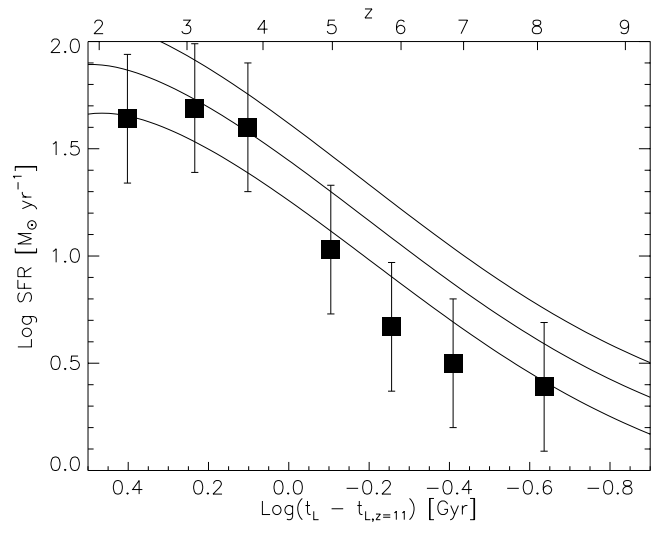

FIG. 10.- Star formation rate in density-selected galaxies as a function of lookback time, normalized to the lookback time at redshift $z=11$. This approximates the evolution of SFR in a growing main-progenitor galaxy. Squares are observations taken from Papovich et al. (2011) selected to have observed number density $n_{0}=2 \times 10^{-4} \mathrm{Mpc}^{-3}$ at all redshifts. Error bars are their suggested values. Lines are values taken from our fiducial model, selected to have number densities $n_{0}=2 \times 10^{-4}, 4 \times 10^{-4}$, and $8 \times 10^{-4} \mathrm{Mpc}^{-3}$, from top to bottom. The smallest value would be appropriate if all the central galaxies were forming stars at a high rate and the Papovich et al. sample is perfectly complete, while the other two values assume that the sampled star-forming galaxies are $50 \%$ or $25 \%$ of the central galaxies. For details on how we derive the model values, see the main text.

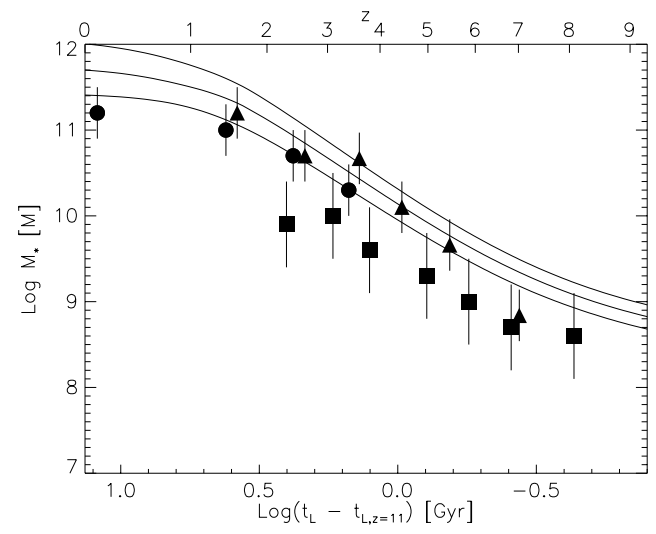

Fig. 11.- Same as Figure 10 but we now plot the stellar mass rather than the star formation rate. Squares again represent UV-selected galaxies. Circles are stellar mass-selected data from Marchesini et al. (2009), and triangles are H-band selected data (which should be similar to stellar mass-selection) from Lundgren et al. (2012, in preparation). The error bars on the UV and stellarmass-selected data sets are the values suggested by Papovich et al. (2011). Data points at very similar lookback times in fact represent samples at the same lookback time, but have been offset from one another for clarity.

ment with the observations. The underlying reason for this trend is the same as for the individual galaxies: at high $z$, stellar mass is roughly proportional to halo mass because most stars are accreted, and star formation rate is also roughly proportional to halo mass because $t_{\mathrm{SF}}$ has only a weak redshift dependence. This provides an explanation for the otherwise puzzling observed sSFR plateau, as discussed in $\$ 1$ and $\$ 5$.

\subsection{The Star-Formation Sequence}

Yet another important observable is the correlation between stellar mass and star formation rate for star form-

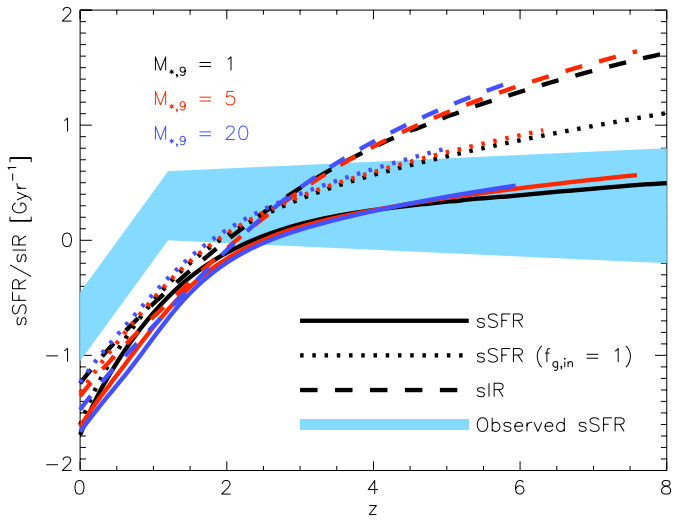

FIG. 12.- Specific star formation rate sSFR $=\dot{M}_{* \text {,form }} / M_{*}$ in our fiducial model (solid lines) and in the $f_{g, \text { in }}=1$ model (dotted lines), and specific gas accretion rate sIR $=\dot{M}_{g, \text { in }} / M_{*}$ (dashed lines) at fixed stellar masses of $M_{*, 9}=1,5$, and 20 (black, red, and blue lines, respectively). The $M_{*, 9}=20$ line does not extend past $z \sim 6$ because even the highest mass of our grid of model halos has not reached this stellar mass at higher redshifts. We do not show the $f_{\mathrm{H}_{2}}=1$ or $M_{\text {ret }}=0.03$ models because they does not differ significantly from the fiducial case in this halo mass range. For comparison, we also show the observed sSFR (shaded region). The region shown is a fit to the data compiled by (Weinmann et al. 2011 see their Figure 1).

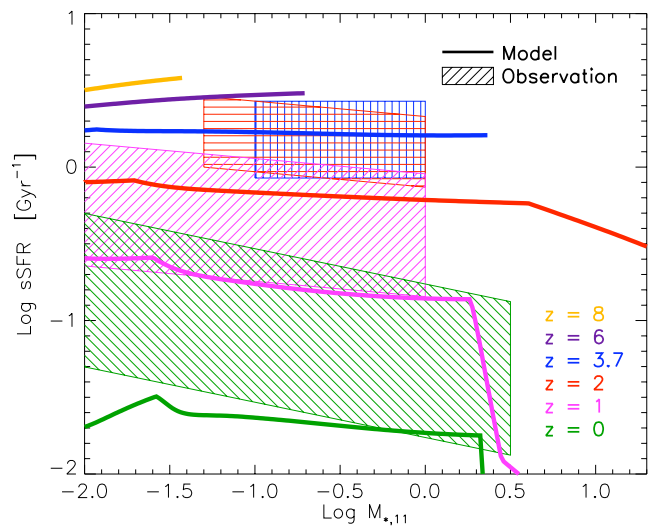

FIG. 13. - The star formation sequence, stellar mass versus specific star formation rate, at $z=0$ (green), $z=1$ (lavender), $z=2$ (red), $z=3.7$ (blue), $z=6$ (indigo), and $z=8$ (orange), from bottom to top, in our model grid (solid lines). Lines end at the maximum stellar mass sampled by our model grid at that redshift. For comparison, hatched regions show the observed correlations at $z=0$ (Brinchmann et al. 2004), $z=1$ (Elbaz et al. 2007), $z=2$ (Daddi et al. 2007), and $z=3.7$ (Lee et al. 2011). For details on how the observed regions are determined, see the main text.

ing galaxies, the "SFR sequence", at different redshifts. This is related to the specific star formation rate versus redshift discussed in Section 4.2, but measured for a range of stellar masses at fixed redshifts rather than for a fixed stellar mass at a range of redshifts.

To extract this quantity from our model grid, we pick a redshift slice to correspond to a chosen observational study, then plot stellar mass versus specific star formation rate for all halos at that redshift. Figure 13 shows the result, compared to several observational surveys: a sample of Lyman Break Galaxies at $z \approx 3.7$ from Lee et al. (2011), a sample of BzK galaxies at $z \approx 2$ from Daddi et al. (2007), a sample of $z \approx 1$ galaxies from 
the GOODS survey studied by Elbaz et al. (2007), and $z \lesssim 0.1$ galaxies from the Sloan Digital Sky Survey studied by Brinchmann et al. (2004). For the Daddi et al. (2007) and Lee et al. (2011) samples, we use the observational constraint regions given in Lee et al. (2011) For the Brinchmann et al. (2004) and Elbaz et al. (2007) samples, we use the fitting formulae given in Elbaz et al. (2007); since they do not give explicit confidence regions or stellar mass ranges, we set our mass range based on eyeball estimates of the region occupied by the data. Similarly, we adopt a scatter of 0.4 dex for the Elbaz et al. (2007) sample and 0.5 dex for the Brinchmann et al. (2004) sample, based on an eyeball estimate of the scatter in the data. We correct all observations to a Chabrier (2005) IMF, which is a factor of 1.59 lower in SFR than a Kennicutt (1998) IMF.

The plot shows that our fiducial model does a reasonably good job of reproducing the observed sSFR sequence, particularly at high redshift. At lower redshift, our models fall slightly below the observed range, consistent with the similar undershoot of sSFR we saw below $z=2$ in Section 4.2 Nonetheless, the level of agreement is gratifying given the extreme simplicity of our model. In particular, we recover the basic results that the sSFR is roughly independent of stellar mass at all redshifts, and that the value of the sSFR varies fairly slowly with redshift above $z \sim 2$. The model also makes clear predictions for the slope and normalization of the SFR sequence at higher redshift than has yet been observed. We do not show the corresponding predictions for our variant model with $f_{g \text {, in }}=1$, but, not surprisingly based on the discussion in Section 4.2 , it provides a much worse fit to the higher redshift data (and a somewhat better fit to the low redshift data). The failure at high redshift is because of the lack of stellar accretion, which produces systematically smaller stellar masses at the same star formation rate.

\subsection{The Mass-Metallicity Relation}

Another observational constraint against which we can compare our model is the mass-metallicity relation. This is not tremendously sensitive to the inclusion or exclusion of metallicity-dependent star formation, because most of the galaxies for which this relation has been observed are in the regime where there is little metallicity quenching. However, it is important as a consistency check. Since the model depends on metallicity affecting the SFR, it is critical that the metallicity evolution the model generates be at least roughly consistent with the observed metallicities of galaxies as a function of mass and redshift.

Such an observational comparison is unfortunately very difficult because of uncertainties in the absolute zero points of the strong-line metallicity indicators that are generally used to infer galaxy metallicities. While the various metallicity measurements in use can be calibrated against each other to a scatter of $\sim 0.03 \mathrm{dex}$, the zero point is uncertain by as much as 0.7 dex (Kewley \& Ellison 2008). Since our models predict an absolute metallicity, i.e. a mass of metals divided by a mass of gas, this means that there will be a large systematic uncertainty in any observational comparison. We handle this problem by fixing the models to match observations at a particular stellar mass and redshift, thereby fixing

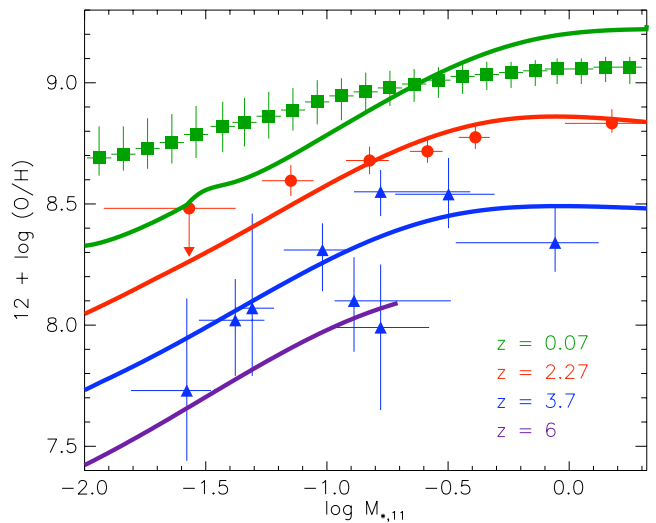

FIG. 14. - Mass-metallicity correlation for our fiducial model (solid lines) at $z=0.07$ (green), $z=2.26$ (red), $z=3.7$ (blue), and $z=6$ (purple), from top to bottom. The $z=3.7$ and $z=6$ lines end at the highest stellar mass contained in our model grid at that redshift. For comparison we show observed masses and metallicities: a sample from Tremonti et al. (2004) at $z=0.07$ (green squares), a sample from Erb et al. (2006) at $z=2.26$ (red circles), and a sample from Maiolino et al. (2008) at $z=3.7$ (blue triangles). The Tremonti et al. (2004) points are averages over many galaxies in the SDSS; the error bars in mass indicate the mass range sampled by each data point, and the error bars in metallicity indicate the range from the 16th to the 84th percentile. The points from Erb et al. (2006) are a sample of UV-selected galaxies; the error bars in mass indicate the standard deviation of the masses contributing to that point, and the error bars in metallicity indicate the errors in the inferred metallicities. The points from Maiolino et al. (2008) are individual galaxies, with the error bars in mass and metalicity indicating the uncertainties in those quantites for each galaxy. The observations have all been corrected to the same IMF and metallicity calibration scheme, and the conversion between metallicity and $12+\log (\mathrm{O} / \mathrm{H})$ from the models has been fixed empirically. See the main text for details. It is important to note that, because of the systematic uncertainties in the strong line calibrations, both the slopes of the various observed relations and the offsets between them should be regarded as highly uncertain.

the zero point. We can then compare the mass and redshift dependence of the models to the observations. It is important to note that we are not looking for more than very rough consistency here, because the slopes of metallicity versus mass and metallicity versus redshift depend on which metallicity calibration one adopts. Different choices of calibration can make a significant difference to how well the data fit the model.

Figure 14 shows a comparison between the massmetallicity relation in our models and a compilation of observations from Tremonti et al. (2004) at $z=0.07$, Erb et al. (2006) at $z=2.27$, and Maiolino et al. (2008) at $z=3.7$. As with other comparisons, we generate the model predictions by finding the redshift slice in our model grid nearest to the target value and plotting mass versus metallicity at that slice. We correct all the observed masses to a Chabrier (2005) IMF, and all the observed metallicities to the KD02 calibration scheme using the conversions of Kewley \& Ellison (2008). We fix the absolute calibration of the models by forcing agreement between the models and the Tremonti et al. (2004) data at $z=0.07$ and $\log M_{*, 11}=-0.5$. This corresponds to adopting a conversion

$$
12+\log (\mathrm{O} / \mathrm{H})=10.75+\log \left(M_{Z} / M_{g}\right),
$$

where $M_{Z}$ and $M_{g}$ are the masses of metals and gas, respectively. 


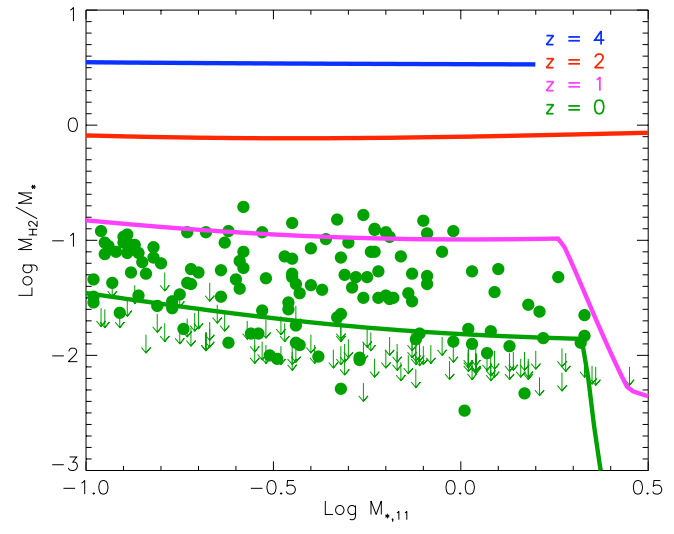

FIG. 15.- Stellar mass versus $\mathrm{H}_{2}$ to stellar mass ratio for our fiducial model (solid lines) at $z=0$ (green), $z=1$ (lavender), $z=2$ (red), and $z=4$ (blue). For comparison, at $z=0$ we show the results of the COLD GASS project (Saintonge et al. 2011a b). Filled circles represent detections, and downward arrows indicate $5 \sigma$ upper limits from non-detections. The high-redshift predictions are to be tested with ALMA.

As the Figure shows, the agreement between the observed and model metallicity evolution is generally good at high $z$. In the present-day universe our model produces a somewhat steeper mass-metallicity relationship than the observed SDSS sample. While this could indicate a real defect in the models, it could equally well be a result of the particular metallicity calibration scheme we have used, since the slope of the SDSS sample (and the other samples) can vary by factors of $\sim 2$ depending on the calibration scheme used. If our models really are too flat compared to reality, this may be related to our models' tendency to somewhat underpredict the star formation rate in small galaxies at low redshift, as we will see in Sections 4.2 and 4.3 . However, since the agreement between our models and the observations is good at $z \gtrsim 2$, our results should be robust there.

\subsection{The $\mathrm{H}_{2}$ Content of Galaxies}

Another feature of our model is its ability to predict the $\mathrm{H}_{2}$ content of galaxies as a function of redshift and other galactic properties. Figure 15 shows the $\mathrm{H}_{2}$ mass fraction versus stellar mass predicted by our models. For comparison, at $z=0$ we show the results of COLD GASS (Saintonge et al.2011a b), a volume-limited survey of CO line emission (used as a proxy to infer $\mathrm{H}_{2}$ ) in several hundred galaxies at distances of $100-200 \mathrm{Mpc}$, selected to have stellar masses $M_{*}>10^{10} M_{\odot}$. As the plot shows, we find generally good agreement between our model and the observed amount of $\mathrm{H}_{2}$ in galaxies at $z=0$, albeit with quite a large spread in the data. We recover the trend that the $\mathrm{H}_{2}$ fraction is (very weakly) declining with stellar mass. We also note that the data contain a significant number of non-detections. Some of these represent star-forming galaxies with relatively modest molecular content whose true value of $M_{\mathrm{H}_{2}} / M_{*}$ is likely only a bit below the reported upper limit. However, some also represent quenched galaxies that are almost entirely devoid of star formation and molecular gas. Our model does not correctly produce galaxies of this type due to our assumption that accretion cuts off sharply at a single, relatively high, halo mass. The observed co-existence of star-forming and passive galaxies at the same stellar (and presumably halo) mass indicates that this is clearly an oversimplification.

At higher redshift, we predict that the $\mathrm{H}_{2}$ fraction at fixed stellar mass should rise, reaching $\sim 10 \%$ by mass at $z=1$ and roughly $50 \%$ by mass at $z=2$. This is qualitatively consistent with the observed trend toward high molecular gas fractions at $z \approx 2$ (e.g. Tacconi et al. 2010), but given the selection biases inherent in the current high $z$ data, it is hard to draw any general conclusions yet. However, these predictions will be testable with ALMA in the next few years (e.g. see reviews by Combes 2010, 2011).

\subsection{Stellar, $\mathrm{H}$ I, and $\mathrm{H}_{2}$ Mass Functions}

Another useful quantity to plot from our models are the mass functions of stars, $\mathrm{H} \mathrm{I}$, and $\mathrm{H}_{2}$ as a function of epoch. Such a comparison can provide some physical insight into the behavior of the model. However, we strongly caution that we should not expect good agreement between the model and observations here. Our models are very simple. Unlike in a full semi-analytic model, we have not made any effort to tune parameters to reproduce observations, and we omit much physics that is known to be necessary to reproduce observed mass functions. For example, we assume that there is one galaxy per halo at all halo masses, clearly not a reasonable assumption for high mass halos that should host clusters. We make the mass loading factor of galactic winds independent of halo mass or other properties, which is probably not reasonable for low masses. Finally, we assume that all halos at a given mass and redshift are the same, ignoring scatter, which is not reasonable given the steepness of the halo mass function.

It is important to note that these omissions are unlikely to dramatically affect the other observational comparisons we present. In particular, we are not particularly concerned with galaxies larger than $L_{*}$, and these do not contribute greatly to the cosmic star formation rate budget, so our incorrect treatment of them is not that important. Similarly, our neglect of scatter can have dramatic effects on the mass function, but it is unlikely to affect correlations between, for example, mass and metallicity. The one factor that could have a significant effect is our assumption of weak galactic winds at low masses, since these can have much the same effect as metallicity quenching in suppressing star formation in small galaxies. In reality both metallicity quenching and strong winds are likely to be important, but we have deliberately kept the winds weak and mass-independent in order to perform a cleaner experiment on the effects of metallicity alone.

To construct our mass functions, we compute the halo abundance $n\left(M_{h}, z\right)$ from the Sheth \& Tormen (1999) approximation as in Section 4.1, and for each halo we assign the $\mathrm{H} \mathrm{I}, \mathrm{H}_{2}$, and stellar mass of the corresponding halo in our grid. Since the stellar mass is a monotonic function of the halo mass in our models, at any redshift $z$ the number density of galaxies $n\left(M_{*}, z\right)$ in the mass range from $M_{*}$ to $M_{*}+d M_{*}$ is simply given by

$$
n\left(M_{*}, z\right)=n\left(M_{h}, z\right) \frac{d M_{*}}{d M_{h}},
$$

where on the right hand side $M_{h}$ is the halo mass for 


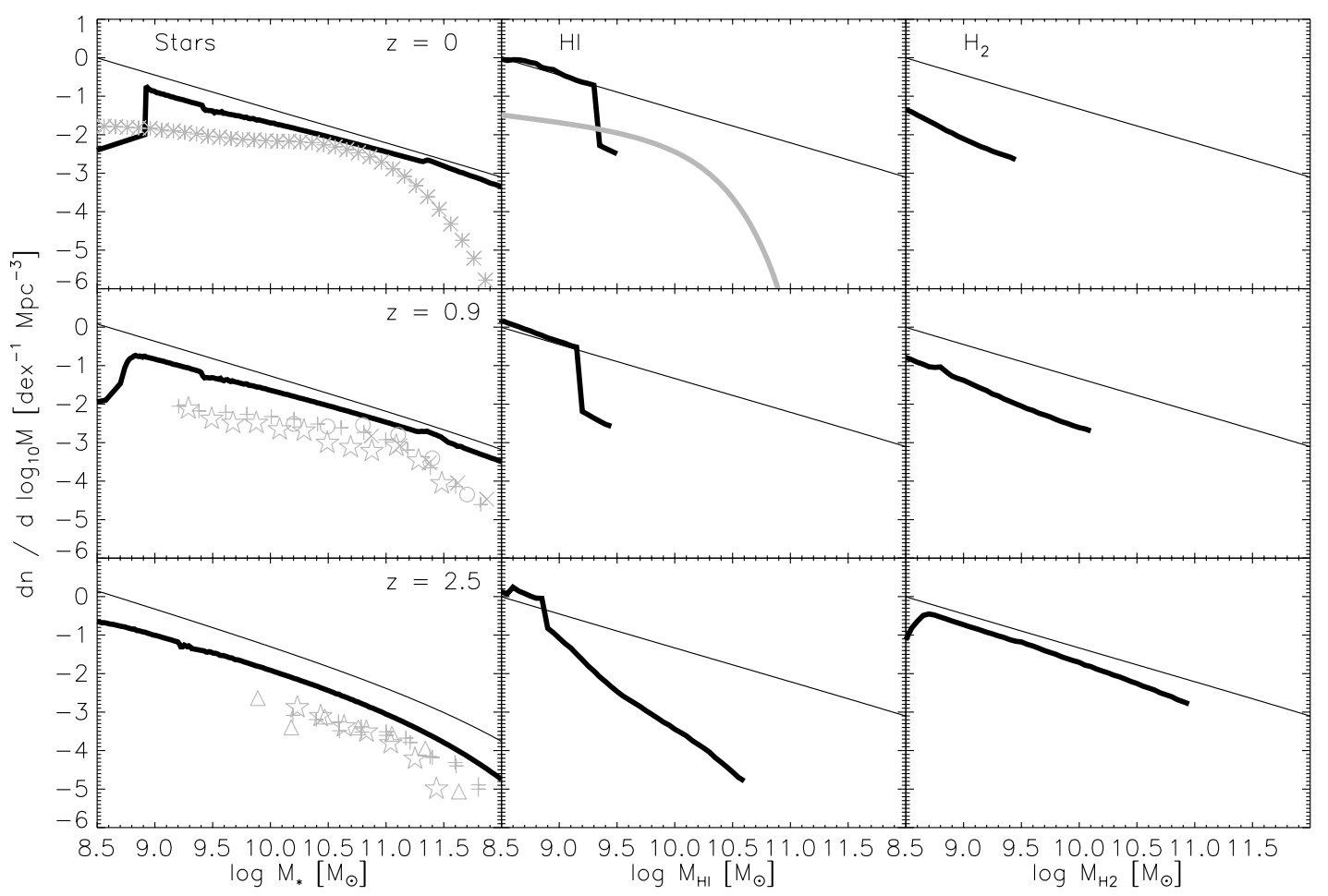

FIG. 16. - Stellar, $\mathrm{H} \mathrm{I}$, and $\mathrm{H}_{2}$ mass functions (left to right columns) at redshifts $z=0, z=0.9$, and $z=2.5$ (top to bottom rows). In each panel, the thick black line is the fiducial model. The thin black line is the mass function that would be produced if all halos had a stellar, $\mathrm{H}$ I, or $\mathrm{H}_{2}$ mass equal to the halo mass times the universal baryon fraction $f_{b}$, e.g. in the left panels the thin black lines show the outcome of baryons accreting onto halos and converting into stars with perfect efficiency, in the middle panels the lines show the result of baryons accreting with perfect efficiency and remaining entirely H I, etc. Gray lines and symbols indicate observations. The stellar mass function points are from Li \& White (2009), as corrected by Guo et al. (2010 $z=0$; asterisks), Bundy et al. (2006 $z=0.75-1$; circles), Borch et al. (2006, $z=0.8-1$; crosses), Pérez-González et al. (2008 $z=0.8-1,2-2.5$, and 2.5 - 3; plusses), Fontana et al. (2006 $z=2-3$; stars), and Marchesini et al. (2009, $z=2-3$; triangles). All stellar data have been corrected to a Chabrier (2005) IMF, and error bars have been omitted tor clarity. The $z=0 \mathrm{H} \mathrm{I}$ mass function is the fit given by Martin et al. (2010). Panels without gray lines or symbols indicate that no data is available.

which the stellar mass is $M_{*}$, and the derivative is evaluated as this mass.

The atomic and molecular gas masses are not strictly monotonic in the halo mass in our model, so there may be multiple halo masses $M_{h, 1}, M_{h, 2}, \ldots$ for which the corresponding atomic or molecular mass is $M_{\mathrm{HI}}$ or $M_{\mathrm{H}_{2}}$. The equivalent expression for the number densities of galaxies with a given $\mathrm{H}$ I mass is therefore

$$
n\left(M_{\mathrm{HI}}, z\right)=\left.\sum_{i} n\left(M_{h, i}, z\right) \frac{d M_{\mathrm{HI}}}{d M_{h}}\right|_{M_{\mathrm{h}, \mathrm{i}}},
$$

where the sum runs over all halo masses $M_{h, i}$ for which the $\mathrm{H}$ I mass is $M_{\mathrm{HI}}$. The expression for $\mathrm{H}_{2}$ is analogous.

Figure 16 shows the model mass functions at three redshifts, compared to data where it is available. The plots reveal several interesting features of the model. First examining the stellar mass function at $z=0$, we see the sharp drop at low stellar masses corresponding to halos where star formation begins to be suppressed by metallicity effects. The mass at which this cutoff occurs moves to smaller values at higher redshifts. Well above this cutoff, our model provides very little suppression of star formation, which is not surprising given that we have not included strong galactic winds or similar mechanisms, and that we do not have any metallicity scatter in out models. Thus we suppress star formation too much below the cutoff mass and too little above it. This shows up in reverse in the $\mathrm{H}$ I mass function, where we overpredict the number of low $\mathrm{H}$ I mass galaxies, and underpredict the number of large H I mass galaxies. Clearly a sharp cutoff in star formation such as the one produced by our model is at best a crude approximation to reality, and the real effects of metallicity should be spread out much more in halo mass.

We also fail to produce galaxies with very large $\mathrm{H}$ I masses, indicating that we are probably converting $\mathrm{H} \mathrm{I}$ to $\mathrm{H}_{2}$ and thence into stars too efficiently in some cases. The origin of this failure is not entirely clear. One possibility is that it comes from our lack of scatter in spin parameter. Galaxies with large H I masses tend to have very large, extended $\mathrm{H}$ I disks that are non-star-forming. Our model does not produce these.

\subsection{The Cosmic Star Formation History}

By combining the star formation rate as a function of halo mass and redshift from our model grid with a calculation of the halo abundance as a function of mass and redshift, we are able to produce the cosmic star formation history for our toy model. We caution that, since the agreement between our simple model and observed sSFRs at redshifts $<2$ is only approximate, we should expect no better agreement here. Our goal is simply to understand the qualitative effect of metallicity quenching on cosmic star formation history with an emphasis 


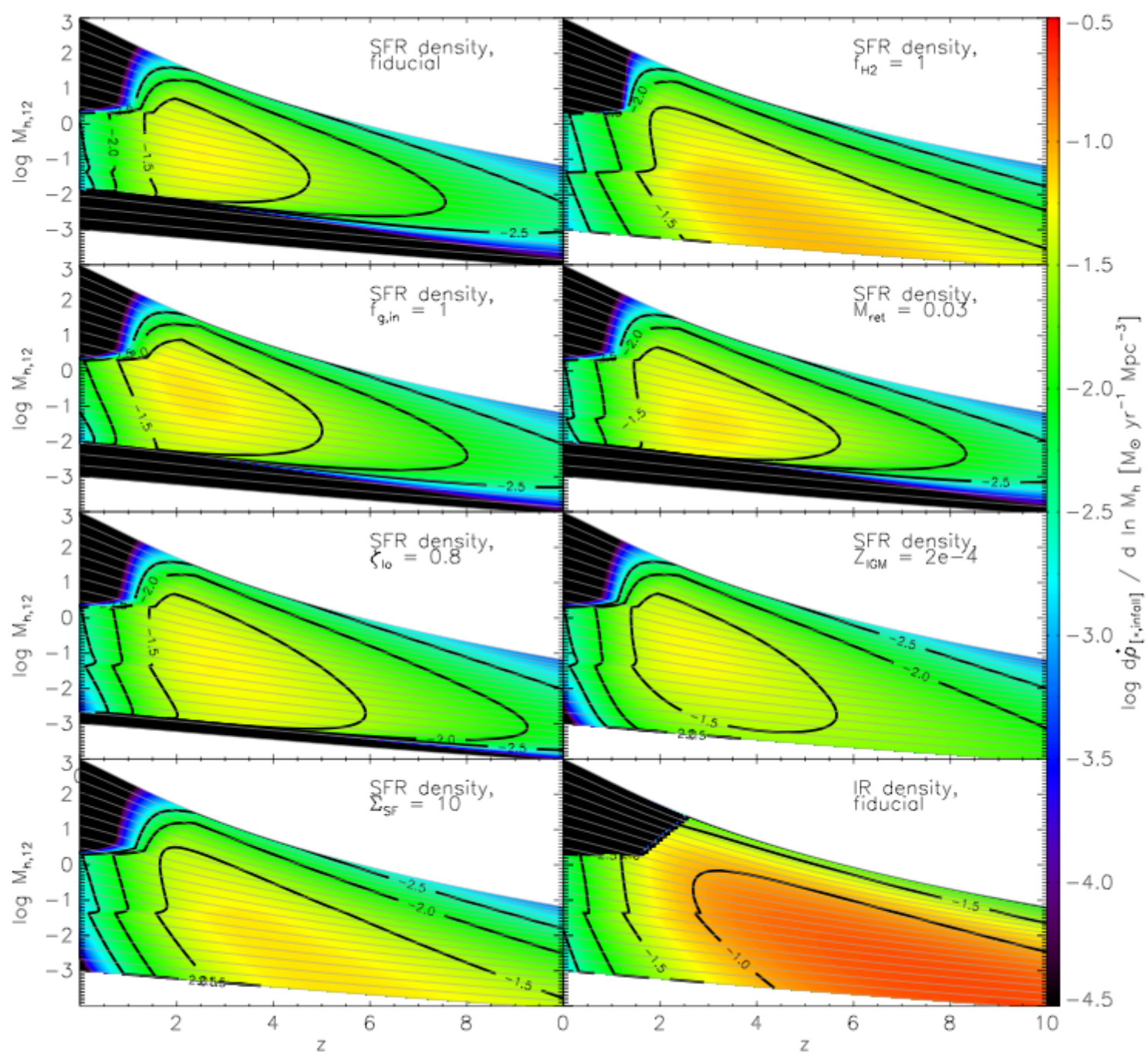

FIG. 17. - The contribution of a halo at a given mass and redshift to the cosmic star formation rate or accretion rate density, $d \dot{\rho}_{[*, \text { acc }]} / d \ln M_{h}=\left[\dot{M}_{*, \text { form }}, \dot{M}_{g, \text { in }}\right]\left(M_{h}, z\right) n\left(M_{h}, z\right) M_{h}$, indicated by color. The format is similar to Figure 7 Heavy black contours show the loci of halos and redshifts with $d \dot{\rho}_{[*, \text { inflow }]} / d \ln M_{h}=-2.5,-2.0,-1.5$, and $-1.0 M_{\odot} \mathrm{yr}^{-1} \mathrm{Mpc}^{-3}$, as indicated.

on the high redshifts where its effects are greatest.

We compute the halo number density $n\left(M_{h}, z\right)$ as in Section 4.1. The cosmic star formation rate density at redshift $z$ is then given by

$$
\dot{\rho}_{*}=\int \dot{M}_{*, \text { form }}\left(M_{h}, z\right) n\left(M_{h}, z\right) M_{h} d \ln M_{h} .
$$

The cosmic density of gas accretion into galaxies is given by the analogous expression with $\dot{M}_{*, \text { form }}$ replaced with $\dot{M}_{g, \text { in }}$. Note that this expression omits stars formed in the accretion shock when $f_{g, \text { in }} \neq 1$, but that is a modest effect.

We plot the integrand of equation (33), and the analogous accretion rate for the cosmic gas accretion density, in Figure 17. The figure illustrates several points. First, our fiducial model range of masses does a good job of sampling the halo masses that dominate the star formation rate, and thus we can make an accurate estimate of $\rho_{*}$ from it for the fiducial model. Second, it is obvious upon inspection that, for the fiducial model, the star for- mation rate density of the universe will peak at $z \sim 2-3$ and fall off sharply on either side of it. On the low $z$ side this fall-off is driven by the decline in star formation rate (which is in turn driven by the falloff in gas accretion rate). On the high $z$ side, the decline in star formation rate is driven by the suppression of star formation due to the difficulty in forming a cold, molecular phase in small halos. In comparison, if we ignore the need to form such a phase and set $f_{\mathrm{H}_{2}}=1$, or if we were to assume that halos could form stars as quickly as they could accrete gas, the cosmic star formation history would be much less peaked toward $z \sim 2-3$. At high redshift it would be dominated by small halos undergoing rapid accretion and star formation.

Figure 18 shows the cosmic star formation rate density as a function of redshift integrated over all halos, both as actually observed and in our variant models, and the cosmic accretion density into halos above a given mass. These latter lines show what we would expect if halos simply turned gas into stars as quickly as they accreted 


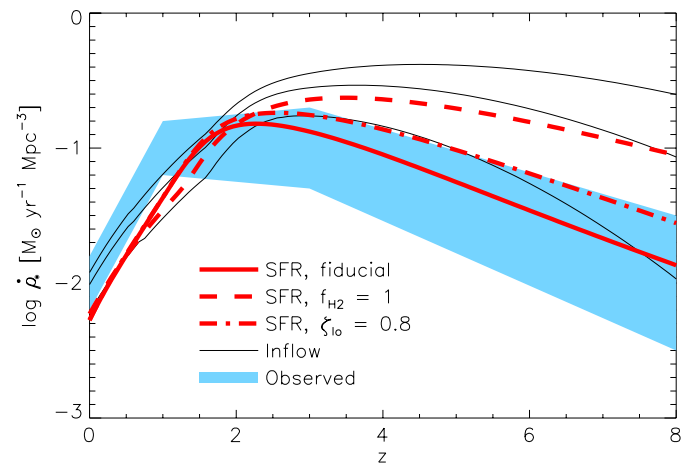

Fig. 18. - Cosmic density of star formation in our fiducial model (solid red line), in the model where we set $f_{\mathrm{H}_{2}}=1$ (dashed red line), in the $\zeta_{\mathrm{lo}}=0.8$ model, and total gas inflow rates into halos with masses above $\log M_{h, 12}=-3,-2$, and -1 (black lines, from top to bottom). We do not show the $f_{g, \text { in }}=1, M_{\text {ret }}=0.03$, or $\Sigma_{\mathrm{SF}}=10$ models, because they differ only slightly from the fiducial model, and we do not show the $Z_{\mathrm{IGM}}=2 \times 10^{-4}$ model because it is qualitatively similar to the $\zeta_{\text {lo }}=0.8$ one. We also show an estimate of the observed cosmic star formation rate density (blue shaded region). The region plotted is a fit based on combining the SFR density estimates compiled by Hopkins \& Beacom (2006), Kistler et al. (2009), Bouwens et al. (2010), and Horiuchi \& Beacom (2010).

it from the IGM; the model with a minimum halo mass $M_{h, 12}=0.1$ is a rough approximation of the model presented by (Bouché et al. 2010), although it is simplified in that it assumes a star formation time of zero as opposed to some number of galactic orbital periods as Bouché et al. assume.

As the plot shows, all the models recover the observed star formation rate from $z=0-2$ reasonably well. Given the long timescales from $z=2$ to the present, the star formation rate in galaxies at this epoch becomes limited only by their gas supply, and it is the decline in gas supply from the $z=2$ to the present that drives this trend. Note that our model SFR is slightly above the best fit observations at $z=2$, but the exact shape and value of the peak in our models depends on how we choose to truncate cold gas accretion in massive halos near $z=2$.

In contrast, the only models that are able to reproduce the observed star formation rate at $z=2-8$ are those that include metallicity quenching (fiducial, $f_{g \text {,in }}=1$ and $\left.M_{\text {ret }}=0.03\right)$ and the ad-hoc model with a threshold for accretion at $M_{h, 12}=0.1$ (as in Bouché et al. 2010). The run with $f_{\mathrm{H}_{2}}=1$ provides a more gradual rise of the SFR density at high redshift, similar to the ad-hoc model with a threshold for accretion at $M_{h, 12}=0.01$, indicating that the suppression because $t_{\mathrm{acc}}$ is shorter than $t_{\mathrm{SF}}$ is effective below a characteristic halo mass of $\sim 10^{10} M_{\odot}$. This is indeed comparable to the Press-Schechter typical halo mass at the end of this period, $z \sim 2$, where the SFR finally catches up with the IR 7 In our fiducial model and its variants that also include metallicity quenching, we recover the steeper rise in time because star formation at high redshift is also suppressed by the difficulty in forming a cold molecular phase in small galaxies that have

\footnotetext{
${ }^{7}$ In fact, the model with $f_{\mathrm{H}_{2}}=1$ should have an even higher star formation rate density at high $z$ than what is plotted, because our model grid does not fully sample the small halos that dominate the star formation rate at high $z$ if we set $f_{\mathrm{H}_{2}}=1$. This is illustrated in Figure 17
}

not yet enriched themselves with metals. The $\zeta_{\text {lo }}=0.8$ model is in between the fiducial model and the $f_{\mathrm{H}_{2}}=1$ model, but is still marginally consistent with the observational constraints. The $Z_{\text {IGM }}=2 \times 10^{-4}$ model (not shown in the plot) is very similar. Thus we see that the amount by which star formation is suppressed when we adopt a realistic treatment of ISM thermodynamics is somewhat but not tremendously sensitive to how metals are retained in halos as a function of mass and to the metal enrichment history of the IGM.

In the simplified model where the star formation rate is set equal to the accretion rate into halos with masses below $M_{h, 12}=0.1$, the mass limit has much the same effect as including metallicity in the star formation law on the integrated star formation rate of the universe. Both suppress star formation at high $z$. This demonstrates that quenching below a threshold mass captures some of the effects of metallicity-dependent star formation. The model presented here provides a physical mechanism that induces a strong but more gradual mass dependence, which boils down to a similar effect on the cosmic SFR history. Our physical model, while it does reduce the SFR in small halos, does not make them entirely devoid of stars, and it still allows galaxies like the Small Magellanic Cloud which lives in a halo of mass $M_{h, 12} \sim 0.01$ (Bekki \& Stanimirović 2009). In reality, the suppression of star formation is probably even less sharp than in our models, since there are almost certainly large stochastic variations in the metal enrichment history of small halos that our simple deterministic model does not capture. The difference between our fiducial model and the $\zeta_{\text {lo }}=0.8$ variant suggests what effect this stochastic variation is likely to have. We should therefore emphasize that the value of the effective threshold mass is predicted by our model only as an order-of-magnitude estimate.

\section{CONCLUSION}

In this paper we investigate how a metallicitydependent star formation rate affects the evolution of star formation in growing galaxies, and how it impacts the observable cosmic star formation history. The physical basis for the effect is that star formation occurs only in the molecular phase of the ISM where the gas is able to reach very low temperatures and low Jeans masses. Because metal atoms and dust grains are the primary coolants and catalysts of $\mathrm{H}_{2}$ formation, and because dust grains are also the primary shield against both photoelectric heating and $\mathrm{H}_{2}$ dissociation by UV photons, the ability of the ISM to form a cold molecular phase depends strongly on its metallicity.

In order to study how these effects modify the star formation histories of galaxies, we have developed an idealized model in which we followed the evolution of the mass and surface density of a galaxy as it accretes baryons, forms stars, and self-enriches with metals. The model is not meant to provide a perfect fit to observations, since we have omitted numerous important processes, but it does allow us to determine qualitatively how the phase transition from warm $\mathrm{H}$ I to cold $\mathrm{H}_{2}$ modifies the observable features of cosmological star formation history. The basic physical model ingredients are:

1. Dark matter and baryons flow into galaxies in an 
average cosmological rate including mergers.

2. Disks have exponential surface density profiles, with scale lengths determined by cosmological evolution under conservation of angular momentum and the assumption that dark matter and baryons share the same spin parameter.

3. Mass is conserved in galaxies as baryons flow in, gas accumulates, turns into stars, and is lost to outflows.

4. The star formation law is that proposed by Krumholz et al. (2009c), in which the SFR per unit area is close to linearly proportional to the column density of the molecular phase of the ISM.

5. The characteristic surface density at which the gas switches to the cold, molecular phase is roughly given by $\Sigma \sim 10 /\left(Z / Z_{\odot}\right)^{-1} M_{\odot} \mathrm{pc}^{-2}$, and near this threshold the cold molecular fraction roughly obeys $f_{\mathrm{H}_{2}} \propto \Sigma\left(Z / Z_{\odot}\right)$.

6. Metal enrichment is a function of the SFR, and the fraction of metals retained in a galaxy rather than lost to the IGM is a function of halo mass.

7. The fraction of ex situ stars in the accretion flow is determined self-consistently over the cosmological halo population.

We find that at very high redshifts, $z>4$, the baryonic mass of the main-progenitor galaxy is growing rapidly with time (roughly $\propto e^{-z}$ ), with an inflow rate that at $z \sim 8$ is already almost as high as at $z \sim 2$. However, at these early times the SFR cannot catch up, because the timescale for star formation in the dominant mode is longer than the timescale for inflow. Furthermore, the low metallicity due to the short history of star formation and the small escape velocity in the typical high- $z$ galaxies is responsible for a low molecular fraction, and this further lowers the SFR compared to the inflow rate. The resultant SFR is growing steeply as $\exp (-0.65 z)$ until $z \sim 2$, contrary to the popular model assuming an exponential decay of SFR. Most of the accreted gas during this period accumulated in an atomic gas reservoir, waiting for the metallicity to grow before it could produce a cold molecular phase and turn into stars.

As time passed in the models, the ratio of timescales for star formation and inflow declined quite rapidly, the forming stars gradually enriched the ISM with metals, and the growing halo mass became more capable of retaining them. The SFR thus grew rapidly, and caught up with the IR by $z \sim 2$. This is consistent with observational findings by Papovich et al. (2010). When integrated over the halo population at a given time, the cosmic SFR density history (SFH) rose with time in the models, in agreement with the observed trend. The inclusion of metallicity in the star formation process affects the SFH in a way that is similar to the effect of a sharp threshold for star formation at halo mass $M_{\mathrm{th}} \sim 10^{11} M_{\odot}$.

We caution that the exact value of the halo mass at which metallicity quenching begins to suppress star formation is not well-determined in our models. It depends on the overall life cycle of metals in galaxies and the IGM, and is sensitive to quantities such as the metal yield of supernovae, the ability of small galaxies to retain the metals they produce, and the metallicity of the IGM gas that accretes onto galaxies at high $z$. Thus our conclusions about how metallicity quenching alters the cosmic star formation history should be regarded as qualitative rather than quantitative. However, the qualitative effect that star formation is suppressed below a threshold mass is robust, and appears in all our models regardless of how we alter the physics of the metal cycle. This has the effect of suppressing star formation in small galaxies at high redshift, and shifting some of this star formation to lower redshift after metals have had a chance to build up. The exact amount of suppression and shifting depends on the exact threshold for metallicity quenching. A threshold of this sort was indeed advocated by Bouché et al. (2010, their Fig. 1) as a possible ad-hoc explanation for the SFH rise, as well as several other phenomena including the downsizing of galaxies (Neistein et al. 2006).

The suppressed in situ SFR makes the stellar fraction in the galaxy small, and the growth of stellar mass dominated by accretion of stars that formed ex situ or in mergers. Under this condition, we showed that the sSFR tends to be roughly constant in time, from very early times till $z \sim 2$. This is consistent with the observational indications for a sSFR plateau for galaxies of a fixed mass observed in the range $z=2-8$. Weinmann et al. (2011) showed that this plateau is rather puzzling when compared with the steeply declining inflow rate with time, but this work shows that including the metallicity dependence helps resolve the issue. One caveat in our otherwise self-consistent model is that we were forced to select a small initial stellar fraction for halos at early times, trying to mimic starbursts in low-metallicity highdensity conditions induced by mergers. Although the existence of a sSFR plateau is not sensitive to this fraction (as long as it is non-zero), the quantitative value and duration of the sSFR plateau is, as described in equation 27. $\mathrm{sSFR} \sim 2\left(f_{*, \text { in }} / 0.1\right)^{-1} \mathrm{Gyr}^{-1}$, where $f_{* \text {,in }} \ll 1$ is the fraction of the inflowing baryons at high $z$ that either arrive already as stars, or that turn into stars in a starburst immediately upon accreting.

We note that the dominance of ex situ over in situ SFR at high redshift is the opposite of what was suggested by the simulations of Oser et al. (2010). While their simulations had efficient SFR in small galaxies at high redshifts, the inclusion of the effects of metallicity on the SFR introduces strong suppression of in situ star formation in halos $\lesssim 10^{11} M_{\odot}$ at $z>2$. This allows a small fraction of ex situ stars to dominate, and naturally lead to a sSFR plateau.

The gas reservoir that builds up at $z \gtrsim 2$ because it cannot form a cold, molecular phase capable of turning into stars provides additional fuel for star formation in $10^{10}-10^{12} M_{\odot}$ halos at $z \sim 1-3$, when the metallicity reaches values high enough to convert the bulk of the ISM to molecules. This allows the SFR to exceed the instantaneous gas IR, which helps to explain the otherwise puzzlingly high SFR density and sSFR observed in this redshift range. The reservoir gas can also help explain the observed massive outflows from $z \sim 2-3$ galaxies (Steidel et al. 2010), which may exceed the inflow rate of fresh gas minus the SFR.

We specify additional observable predictions of our model, as a function of galaxy stellar mass and redshift. 
First, the sSFR sequence, of sSFR versus stellar mass, is rather flat below $M_{*} \sim 10^{11.5} M_{\odot}$ at all redshifts. This is complementary to the constancy of the sSFR with time at $z>2$. Second, at any redshift, the metallicity is rising roughly $\propto M_{*}^{1 / 2}$ in the range $M_{*}<10^{10.5} M_{\odot}$, and it flattens off at higher masses. At a given stellar mass, in the range $z \geq 2$, the metallicity grows in time roughly $\propto z^{-3}$. Third, the $\mathrm{H}_{2}$ fraction is very weakly decreasing with mass at fixed redshift, and is higher at higher redshifts. At $z \approx 2, \mathrm{H}_{2}$ masses are roughly equal to galactic stellar masses. We should add that the slow SFR and the resultant gas accumulation at high redshift is likely to lead to extended discs with low bulge-to-disk ratios, and thus help explain one of the most interesting open questions in galaxy formation (Guedes et al. 2011, Brook et al. 2011).

We thank the referee for a helpful report. We acknowledge stimulating discussions with R. Bouwens, F. Combes, M. Kuhlen, K. Noeske, and A. Hopkins. We thank S. Weinmann for providing the data compilation used in Figure 16. MRK acknowledges support from: an Alfred P. Sloan Fellowship; the National Science Foundation through grants AST-0807739 and CAREER-0955300; and NASA through Astrophysics Theory and Fundamental Physics grant NNX09AK31G, a Spitzer Space Telescope theoretical research program grant, and a Chandra Space Telescope grant. AD acknowledges support from: ISF grant 6/08, GIF grant G-1052-104.7/2009, a DIP grant, and NSF grant AST1010033 .

\section{APPENDIX}

\section{A. PARAMETERS FOR GAS EXPULSION AND STELLAR RECYCLING AND YIELD}

We adopt $\epsilon_{\text {out }}=1.0$, based on the estimates of Erb $(2008)$ that galactic winds tend to carry mass fluxes comparable to the galactic star formation rate. In reality the wind mass loading factor almost certainly depends on galactic properties such as the halo circular velocity, star formation rate, or star formation rate surface density. We do not attempt to include this dependence. In part this is because it is extremely poorly determined both observationally and theoretically. However, experimenting with different values of $\epsilon_{\text {out }}$, including mass-dependent ones, shows that is has very little effect on metallicity quenching. Halos that are small enough to have their star formation quenched by metallicity effects do not form many stars, and thus it matters fairly little what their mass loading factor is. Conversely, since galactic winds that do not preferentially carry away metals (i.e. those described by $\epsilon_{\text {out }}$ rather than $\zeta$ in our formalism) do not alter the metallicity, the choice of $\epsilon_{\text {out }}$ changes the absolute stellar mass of large halos, but it does not change the extent to which they are affected by metallicity quenching.

For $R$ and $y$, following the notation of Tinsley (1980), we define the return fraction by

$$
R=\frac{\int_{m_{l}}^{m_{u}}[m-w(m)] \phi(m) d m}{\int_{m_{l}}^{m_{u}} m \phi(m) d m},
$$

where $\phi(m)$ is the stellar IMF, $w(m)$ is the mass of the remnant left by a star of mass $m$, and $m_{l}$ and $m_{u}$ are the lower and upper mass limits of the IMF. The yield of element $i$ is defined by

$$
y_{i}=\frac{1}{1-R} \frac{\int_{m_{l}}^{m_{u}} m p_{i}(m) \phi(m) d m}{\int_{m_{l}}^{m_{u}} m \phi(m) d m}
$$

where $p_{i}(m)$ is the net fraction of the star's initial mass that is converted to element $i$ in a star of mass $m$.

We adopt the instantaneous recycling approximation, and set

$$
w(m)=\left\{\begin{array}{ll}
m, & m / M_{\odot} \leq 1 \\
0.7 M_{\odot}, & 1<m / M_{\odot} \leq 8 \\
1.4 M_{\odot}, & m / M_{\odot}>8
\end{array},\right.
$$

corresponding to an assumption that stars of mass $m<1.0 M_{\odot}$ never leave the main sequence, those of mass $m=1-8$ $M_{\odot}$ produce white dwarfs of mass $0.7 M_{\odot}$, and those of mass $m>8 M_{\odot}$ produce $1.4 M_{\odot}$ neutron stars. For $\phi(m)$ we use the Chabrier (2005) form,

$$
\phi(m)= \begin{cases}0.41 m^{-1} \exp \left[-\frac{(\log m-\log 0.2)^{2}}{2 \times(0.55)^{2}}\right], & m \leq 1 M_{\odot} \\ 0.18 m^{-2.35} & m>1 M_{\odot}\end{cases}
$$

within the range $m_{l}=0.08 M_{\odot}, m_{u}=120 M_{\odot}$. With this IMF and value of $w(m)$, we have $R=0.46$.

For the production function $p_{i}(m)$, we adopt the models of Maeder $(1992)$ for $Z=0.001$ (i.e. low metallicity) stars (his Table 5). Since we are treating metallicity with a single parameter, we use the total metal yields, and interpolate between the tabulated values. With these models and our chosen IMF, the yield is $y=0.069$. If we instead use the models for Solar metallicity, $y=0.054$ instead, and the change in results is small. We prefer to use the low-metallicity value because our models are most sensitive to the metallicity evolution when the overall metallicity is small.

One might worry that instantaneous recycling becomes a poor approximation in the early universe, where stellar lifetimes are not necessarily short compared to the Hubble time. However, the approximation is surprisingly good. Using starburst99 (Leitherer et al. 1999, Vázquez \& Leitherer 2005) for a Kroupa (2002) IMF with an upper mass 
cutoff of $120 M_{\odot}$, we find that the return fraction reaches $R=0.27$ after only 100 Myr, i.e. about $60 \%$ of the mass that our instantaneous recycling approximation predicts will eventually be returned to the ISM is returned in the first $100 \mathrm{Myr}$ after star formation. Metal production is even faster. For comparison, at $z=30$, the redshift at which we begin our calculations, the age of the universe for our cosmological parameters is also very close to 100 Myr. Thus the error made by the instantaneous recycling approximation is at most a factor of $\sim 2$ very early in our calculations, and becomes significantly smaller than that even by redshift 10 .

\section{B. COMPUTING THE GASEOUS INFLOW FRACTION}

The gaseous inflow fraction $f_{g \text {,in }}$ is expected to depend on the halo mass $M_{h}$ and redshift $z$. We estimate $f_{g \text {,in }}$ selfconsistently using the grid of models we describe in Section 3. The procedure is as follows. First, we must estimate

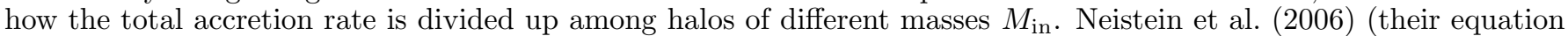
A15) show that the total accretion rate onto a halo of mass $M_{h}$ arising from halos of mass less than $M_{\text {in }}$ may be approximated by

$$
\dot{M}_{h}=-\sqrt{\frac{2}{\pi}} \frac{M_{h}}{\sqrt{S\left(M_{\text {in }}\right)-S\left(M_{h}\right)}} \dot{\omega},
$$

where $S(M)=\sigma^{2}(M)$ is the variance of the density fluctuation in top-hat spheres containing average mass $M$; we approximate this using the fitting function given in equation (A16) of Neistein et al. (2006). The total accretion rate is given by Equation (B1) evaluated with $M_{\text {in }} \approx M_{h} / 2$, and the differential contribution to the accretion rate by halos in the mass range $M_{\text {in }}$ to $M_{\text {in }}+d M_{\text {in }}$ is given by

$$
\frac{d \dot{M}_{h}}{d M_{\mathrm{in}}}=\sqrt{\frac{1}{2 \pi}} \frac{M_{h}}{\left[S\left(M_{\mathrm{in}}\right)-S\left(M_{h}\right)\right]^{3 / 2}} \frac{d S\left(M_{\mathrm{in}}\right)}{d M_{\mathrm{in}}} \dot{\omega} .
$$

Note that $\dot{M}_{h}$ is non-zero even for $M_{\text {in }}=0$, because some accretion is contributed by matter that is not in an existing halo (see also Genel et al. 2010). We define the non-halo accretion rate

$$
\dot{M}_{h, \text { non-halo }}=\dot{M}_{h}\left(M_{\text {in }}=0\right) \text {. }
$$

Numerical evaluation shows that $\dot{M}_{h, \text { non-halo }} / \dot{M}_{h} \sim 0.2$, decreasing very weakly with $M_{h}$. Given these expressions, we can approximate $f_{g \text {,in }}$ for a given halo by computing the accretion-weighted mean gas fraction of all the halos that are accreting, i.e.

$$
f_{g, \text { in }} \approx \frac{1}{\dot{M}_{h}}\left[\int_{0}^{M_{h} / 2} f_{g}\left(M_{\mathrm{in}}\right) \frac{d \dot{M}_{h}}{d M_{\mathrm{in}}} d M_{\mathrm{in}}+\dot{M}_{h, \text { non-halo }}\right] .
$$

Note that we have assumed here that the baryonic component of the non-halo accretion is pure gas.

In practice, we evaluate Equation (B4) numerically as follows. We assume that there is no star formation in halos smaller than the smallest halo in our grid of 400 model halos (which is true at least for our fiducial model), and so we adopt $f_{g}\left(M_{\text {in }}\right)=f_{g \text {,init }}$ for all values of $M_{\text {in }}$ smaller than the smallest halo in our model grid. Thus, the stellar fraction of these halos never changes. For larger values of $M_{\mathrm{in}}$, we store our grid of models at 400 redshifts from 30 (our starting redshift) to 0 , and evaluate $f_{g}$ by interpolating on the grid in halo mass and redshift. This procedure does not require any iteration to converge, because for a halo of mass $M_{h}$ at a given $z$, we only need to evaluate $f_{g}\left(M_{\text {in }}\right)$ for values of $M_{\text {in }}<M_{h} / 2$. Provided that we compute the evolution of our model grid starting with the smallest halo and proceeding upward in mass, by the time we reach a halo of a given mass, we already know the full evolutionary history of all lower mass halos, and thus those parts of the interpolation grid we require have already been filed in.

\section{REFERENCES}

Abel, T., Bryan, G. L., \& Norman, M. L. 2002, Science, 295, 93 Barnes, L. A., \& Haehnelt, M. G. 2010, MNRAS, 403, 870

Baugh, C. M., Lacey, C. G., Frenk, C. S., Granato, G. L., Silva, L., Bressan, A., Benson, A. J., \& Cole, S. 2005, MNRAS, 356, 1191

Bekki, K., \& Stanimirović, S. 2009, MNRAS, 395, 342

Bigiel, F., Leroy, A., Walter, F., Blitz, L., Brinks, E., de Blok, W. J. G., \& Madore, B. 2010, AJ, 140, 1194

Bigiel, F., Leroy, A., Walter, F., Brinks, E., de Blok, W. J. G., Madore, B., \& Thornley, M. D. 2008, AJ, 136, 2846

Birnboim, Y., \& Dekel, A. 2003, MNRAS, 345, 349

Birnboim, Y., Dekel, A., \& Neistein, E. 2007, MNRAS, 380, 339

Blumenthal, G. R., Faber, S. M., Primack, J. R., \& Rees, M. J. 1984, Nature, 311, 517

Bolatto, A. D., et al. 2011, ApJ, submitted

-. 2007, ApJ, 655, 212

Borch, A., et al. 2006, A\&A, 453, 869

Bouché, N., et al. 2010, ApJ, 718, 1001

Bouwens, R. J., et al. 2010, ApJ, 709, L133
Brinchmann, J., Charlot, S., White, S. D. M., Tremonti, C., Kauffmann, G., Heckman, T., \& Brinkmann, J. 2004, MNRAS, 351,1151

Bromm, V., Coppi, P. S., \& Larson, R. B. 2002, ApJ, 564, 23

Brook, C. B., et al. 2011, MNRAS, 415

Bullock, J. S., Dekel, A., Kolatt, T. S., Kravtsov, A. V., Klypin,

A. A., Porciani, C., \& Primack, J. R. 2001, ApJ, 555, 240

Bundy, K., et al. 2006, ApJ, 651, 120

Burkert, A., et al. 2010, ApJ, 725, 2324

Cattaneo, A., Dekel, A., Devriendt, J., Guiderdoni, B., \& Blaizot, J. 2006, MNRAS, 370, 1651

Cen, R., \& Ostriker, J. P. 2006, ApJ, 650, 560

Chabrier, G. 2005, in Astrophysics and Space Science Library, Vol. 327, The Initial Mass Function 50 Years Later, ed. E. Corbelli, F. Palla, \& H. Zinnecker, 41-+

Cirasuolo, M., McLure, R. J., Dunlop, J. S., Almaini, O., Foucaud, S., \& Simpson, C. 2010, MNRAS, 401, 1166

Combes, F. 2010, Highlights of Astronomy, 15, 418 
Combes, F. 2011, in IAU Symposium 277, ed. C. Carignan, F. Combes, \& K. Freeman, in press, arXiv:1101.3077

Conroy, C., \& Wechsler, R. H. 2009, ApJ, 696, 620

Conroy, C., Wechsler, R. H., \& Kravtsov, A. V. 2006, ApJ, 647, 201

Croxall, K. V., van Zee, L., Lee, H., Skillman, E. D., Lee, J. C., Côté, S., Kennicutt, Jr., R. C., \& Miller, B. W. 2009, ApJ, 705, 723

Daddi, E., et al. 2007, ApJ, 670, 156

Davé, R. 2011, in Astronomical Society of the Pacific Conference Series, Vol. 440, Astronomical Society of the Pacific Conference Series, ed. M. Treyer, T. Wyder, J. Neill, M. Seibert, \& J. Lee, $353-+$

Dekel, A., \& Birnboim, Y. 2006, MNRAS, 368, 2

Dekel, A., et al. 2009, Nature, 457, 451

Dekel, A., \& Silk, J. 1986, ApJ, 303, 39

Dutton, A. A., et al. 2010, MNRAS, 1493

Efstathiou, G. 1992, MNRAS, 256, 43P

Elbaz, D., et al. 2007, A\&A, 468, 33

Erb, D. K. 2008, ApJ, 674, 151

Erb, D. K., Shapley, A. E., Pettini, M., Steidel, C. C., Reddy, N. A., \& Adelberger, K. L. 2006, ApJ, 644, 813

Faucher-Giguere, C., Keres, D., \& Ma, C. 2011, MNRAS, submitted, arXiv: 1103.0001

Fontana, A., et al. 2006, A\&A, 459, 745

Fontanot, F., De Lucia, G., Monaco, P., Somerville, R. S., \& Santini, P. 2009, MNRAS, 397, 1776

Fu, J., Guo, Q., Kauffmann, G., \& Krumholz, M. R. 2010, MNRAS, 409, 515

Fumagalli, M., Krumholz, M. R., \& Hunt, L. K. 2010, ApJ, 722, 919

Genel, S., Bouché, N., Naab, T., Sternberg, A., \& Genzel, R. 2010, ApJ, 719, 229

Genzel, R., et al. 2008, ApJ, 687, 59

-. 2011, ApJ, 733, 101

-. 2006, Nature, 442, 786

Glover, S. C. O., \& Clark, P. C. 2011, MNRAS, submitted, arXiv: 1105.3073

Gnedin, N. Y., \& Kravtsov, A. V. 2010, ApJ, 714, 287

Gnedin, N. Y., Tassis, K., \& Kravtsov, A. V. 2009, ApJ, 697, 55

González, V., Labbé, I., Bouwens, R. J., Illingworth, G., Franx, M., Kriek, M., \& Brammer, G. B. 2010, ApJ, 713, 115

Governato, F., Willman, B., Mayer, L., Brooks, A., Stinson, G., Valenzuela, O., Wadsley, J., \& Quinn, T. 2007, MNRAS, 374, 1479

Guedes, J., Callegari, S., Madau, P., \& Mayer, L. 2011, ApJ, submitted, arXiv: 1103.6030

Guo, Q., White, S., Li, C., \& Boylan-Kolchin, M. 2010, MNRAS, 404,1111

Hernquist, L., \& Springel, V. 2003, MNRAS, 341, 1253

Hoeft, M., Yepes, G., Gottlöber, S., \& Springel, V. 2006, MNRAS, 371, 401

Hopkins, A. M., \& Beacom, J. F. 2006, ApJ, 651, 142

Hopkins, P. F., Quataert, E., \& Murray, N. 2011, MNRAS, 1513 , in press, arXiv:1101.4940

Horiuchi, S., \& Beacom, J. F. 2010, ApJ, 723, 329

Kennicutt, Jr., R. C. 1998, ApJ, 498, 541

Kennicutt, Jr., R. C., et al. 2007, ApJ, 671, 333

Kereš, D., Katz, N., Davé, R., Fardal, M., \& Weinberg, D. H. 2009a, MNRAS, 396, 2332

Kereš, D., Katz, N., Fardal, M., Davé, R., \& Weinberg, D. H. 2009b, MNRAS, 395, 160

Kereš, D., Katz, N., Weinberg, D. H., \& Davé, R. 2005, MNRAS, 363,2

Kewley, L. J., \& Ellison, S. L. 2008, ApJ, 681, 1183

Kistler, M. D., Yüksel, H., Beacom, J. F., Hopkins, A. M., \& Wyithe, J. S. B. 2009, ApJ, 705, L104

Kobayashi, C. 2004, MNRAS, 347, 740

Kroupa, P. 2002, in ASP Conf. Ser. 285: Modes of Star Formation and the Origin of Field Populations, ed. E. K. Grebel \& W. Brandner, 86

Krumholz, M. R., Ellison, S. L., Prochaska, J. X., \& Tumlinson, J. 2009a, ApJ, 701, L12

Krumholz, M. R., \& Gnedin, N. Y. 2011, ApJ, 729, 36

Krumholz, M. R., Leroy, A. K., \& McKee, C. F. 2011, ApJ, 731, 25
Krumholz, M. R., McKee, C. F., \& Tumlinson, J. 2008, ApJ, 689, 865

-. 2009b, ApJ, 693, 216

-. 2009c, ApJ, 699, 850

Krumholz, M. R., \& Tan, J. C. 2007, ApJ, 654, 304

Krumholz, M. R., \& Thompson, T. A. 2007, ApJ, 669, 289

Kuhlen, M., Krumholz, M., Madau, P., Smith, B., \& Wise, J. 2011, ApJ, submitted, arXiv:1105.2376

Lagos, C. d. P., Lacey, C. G., Baugh, C. M., Bower, R. G., \& Benson, A. J. 2010, MNRAS, submitted, arXiv:1011.5506

Lee, K.-S., et al. 2011, ApJ, 733, 99

Leitherer, C., et al. 1999, ApJS, 123, 3

Leroy, A., Bolatto, A., Stanimirovic, S., Mizuno, N., Israel, F., \& Bot, C. 2007, ApJ, 658, 1027

Leroy, A. K., Walter, F., Brinks, E., Bigiel, F., de Blok, W. J. G., Madore, B., \& Thornley, M. D. 2008, AJ, 136, 2782

Li, C., \& White, S. D. M. 2009, MNRAS, 398, 2177

Loeb, A., \& Barkana, R. 2001, ARA\&A, 39, 19

Mac Low, M., \& Ferrara, A. 1999, ApJ, 513, 142

Maeder, A. 1992, A\&A, 264, 105

Maiolino, R., et al. 2008, A\&A, 488, 463

Marchesini, D., van Dokkum, P. G., Förster Schreiber, N. M., Franx, M., Labbé, I., \& Wuyts, S. 2009, ApJ, 701, 1765

Martin, A. M., Papastergis, E., Giovanelli, R., Haynes, M. P., Springob, C. M., \& Stierwalt, S. 2010, ApJ, 723, 1359

McKee, C. F., \& Krumholz, M. R. 2010, ApJ, 709, 308

Mo, H. J., \& White, S. D. M. 2002, MNRAS, 336, 112

Murali, C., Katz, N., Hernquist, L., Weinberg, D. H., \& Davé, R. 2002, ApJ, 571, 1

Murray, N., Quataert, E., \& Thompson, T. A. 2010, ApJ, 709, 191

Neistein, E., \& Dekel, A. 2008, MNRAS, 383, 615

Neistein, E., van den Bosch, F. C., \& Dekel, A. 2006, MNRAS, 372,933

Obreschkow, D., Croton, D., De Lucia, G., Khochfar, S., \&

Rawlings, S. 2009, ApJ, 698, 1467

Obreschkow, D., \& Rawlings, S. 2009, ApJ, 696, L129

Ocvirk, P., Pichon, C., \& Teyssier, R. 2008, MNRAS, 390, 1326

Oey, M. S., \& Kennicutt, Jr., R. C. 1993, ApJ, 411, 137

Oppenheimer, B. D., \& Davé, R. 2006, MNRAS, 373, 1265

Oser, L., Ostriker, J. P., Naab, T., Johansson, P. H., \& Burkert, A. 2010, ApJ, 725, 2312

Papovich, C., Finkelstein, S. L., Ferguson, H. C., Lotz, J. M., \&

Giavalisco, M. 2011, MNRAS, 412, 1123

Papovich, C., et al. 2010, ApJ, 716, 1503

-. 2006, ApJ, 640, 92

Pérez-González, P. G., et al. 2008, ApJ, 675, 234

Rafelski, M., Wolfe, A. M., \& Chen, H. 2010, ArXiv e-prints

Robertson, B. E., \& Kravtsov, A. V. 2008, ApJ, 680, 1083

Rodighiero, G., et al. 2011, ApJ, 739, L40

Saintonge, A., et al. 2011a, MNRAS, 415, 32

-. 2011b, MNRAS, 61

Schaye, J. 2004, ApJ, 609, 667

Schaye, J., Aguirre, A., Kim, T.-S., Theuns, T., Rauch, M., \&

Sargent, W. L. W. 2003, ApJ, 596, 768

Schaye, J., et al. 2010, MNRAS, 402, 1536

Shaviv, N. J., \& Dekel, A. 2003, ArXiv Astrophysics e-prints, arXiv:astro-ph/0305527

Sheth, R. K., \& Tormen, G. 1999, MNRAS, 308, 119

Simcoe, R. A. 2011, ApJ, 738, 159

Springel, V., \& Hernquist, L. 2003, MNRAS, 339, 312

Stark, D. P., Ellis, R. S., Bunker, A., Bundy, K., Targett, T., Benson, A., \& Lacy, M. 2009, ApJ, 697, 1493

Steidel, C. C., Erb, D. K., Shapley, A. E., Pettini, M., Reddy, N., Bogosavljević, M., Rudie, G. C., \& Rakic, O. 2010, ApJ, 717, 289

Tacconi, L. J., et al. 2010, Nature, 463, 781

Thompson, T. A., Quataert, E., \& Murray, N. 2005, ApJ, 630, 167

Thoul, A. A., \& Weinberg, D. H. 1996, ApJ, 465, 608

Tinsley, B. M. 1980, Fundamentals of Cosmic Physics, 5, 287

Tremonti, C. A., et al. 2004, ApJ, 613, 898

Tumlinson, J., et al. 2002, ApJ, 566, 857

van Dokkum, P. G., et al. 2008, ApJ, 677, L5

Vázquez, G. A., \& Leitherer, C. 2005, ApJ, 621, 695

Weinmann, S. M., Neistein, E., \& Dekel, A. 2011, MNRAS, 417, 2737 
Werk, J. K., Putman, M. E., Meurer, G. R., \& Santiago-Figueroa, N. 2011, ApJ, 735, 71

Wild, V., Hewett, P. C., \& Pettini, M. 2007, MNRAS, 374, 292

Williams, B. F., Dalcanton, J. J., Dolphin, A. E., Holtzman, J., \& Sarajedini, A. 2009, ApJ, 695, L15

Wise, J. H., Turk, M. J., Norman, M. L., \& Abel, T. 2010, ApJ, submitted, arXiv:1011.2632
Wolfe, A. M., \& Chen, H.-W. 2006, ApJ, 652, 981

Wong, T., \& Blitz, L. 2002, ApJ, 569, 157

Wyder, T. K., et al. 2009, ApJ, 696, 1834 


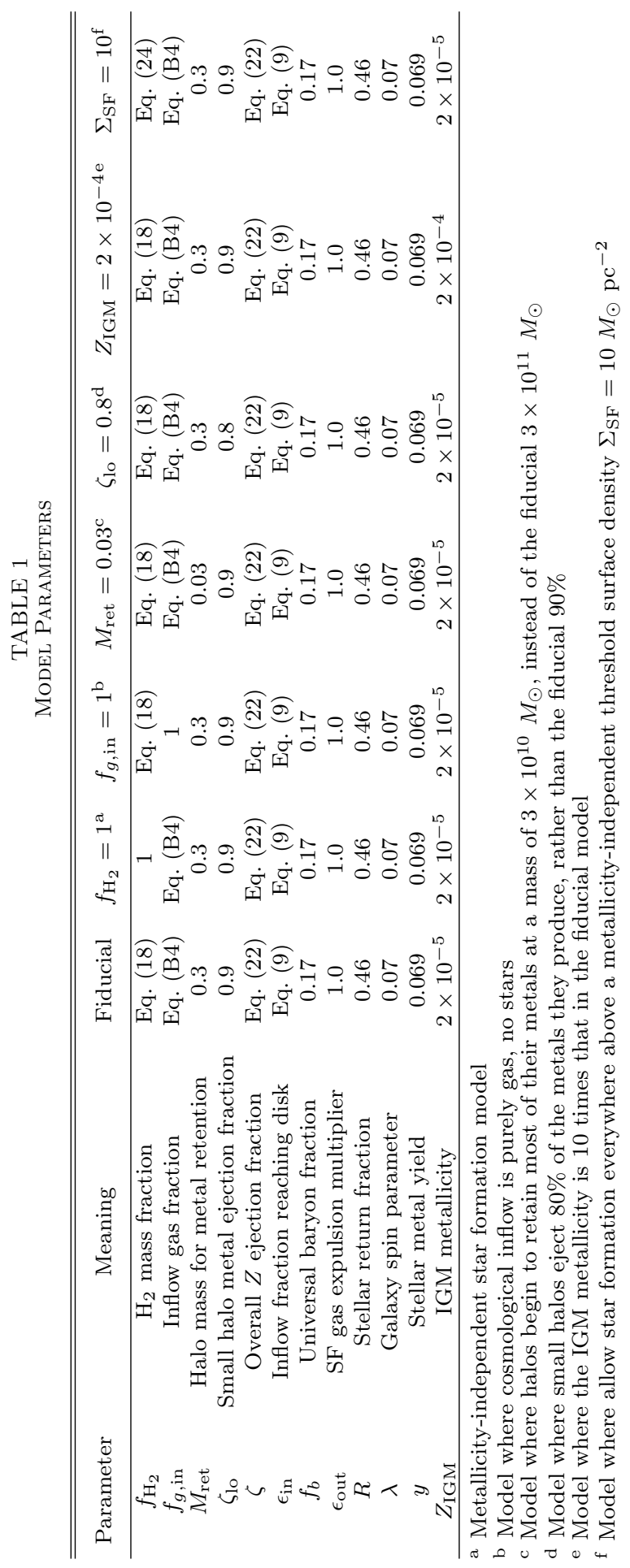

